\title{
A neural mechanism for coordinate transformation predicts pre-saccadic remapping
}

\author{
Sebastian Schneegans • Gregor Schöner
}

Received: 16 August 2011 / Accepted: 13 March 2012 / Published online: 6 April 2012

(c) The Author(s) 2012. This article is published with open access at Springerlink.com

\begin{abstract}
Whenever we shift our gaze, any location information encoded in the retinocentric reference frame that is predominant in the visual system is obliterated. How is spatial memory retained across gaze changes? Two different explanations have been proposed: Retinocentric information may be transformed into a gaze-invariant representation through a mechanism consistent with gain fields observed in parietal cortex, or retinocentric information may be updated in anticipation of the shift expected with every gaze change, a proposal consistent with neural observations in LIP. The explanations were considered incompatible with each other, because retinocentric update is observed before the gaze shift has terminated. Here, we show that a neural dynamic mechanism for coordinate transformation can also account for retinocentric updating. Our model postulates an extended mechanism of reference frame transformation that is based on bidirectional mapping between a retinocentric and a bodycentered representation and that enables transforming multiple object locations in parallel. The dynamic coupling between the two reference frames generates a shift of the retinocentric representation for every gaze change. We account for the predictive nature of the observed remapping activity by using the same kind of neural mechanism to generate an internal representation of gaze direction that is predictively updated based on corollary discharge signals. We provide evidence for the model by accounting for a series of behavioral and neural experimental observations.
\end{abstract}

Keywords Saccadic eye movements - Spatial memory · Gain modulation · Neural field model $\cdot$ Lateral intraparietal cortex (LIP) · Frontal eye field (FEF)

S. Schneegans $(\varangle) \cdot$ G. Schöner

Institut für Neuroinformatik, Ruhr-Universität Bochum,

44780 Bochum, Germany

e-mail: sebastian.schneegans@ini.rub.de

\section{Introduction}

When visually exploring the environment, humans and other primates frequently shift their gaze in order to foveate regions of interest. Each gaze shift alters the reference frame of visual perception, and therefore the relationship between the position of an image on the retina and the position of the corresponding object in the world. Consequently, memorizing the retinal position of a perceived object is not sufficient by itself to retrieve its location after a gaze change. Yet, it is clear that primates possess trans-saccadic memory of locations as evident from experiments in the double-step saccade tasks (Hallett and Lightstone 1976). To explain this ability, two hypotheses have been proposed: According to the first one, the spatial information that is initially in a retinocentric reference frame is combined with information on the current gaze direction and transformed into a gaze-invariant representation (Mays and Sparks 1980). The second hypothesis holds that a purely retinocentric representation is used, which is internally updated for every gaze change such that it remains aligned with the visual input (Duhamel et al. 1992).

In support of the first hypothesis, Andersen and colleagues identified a possible neural mechanism for the reference frame transformation required therein (Andersen and Mountcastle 1983; Andersen et al. 1985): They found neurons in the posterior parietal cortex which have visual receptive fields in eye-centered coordinates, but whose overall response strength is modulated by the current eye position. Similar response properties have also been described for neurons in the frontal eye field (FEF, Cassanello and Ferrera 2007). In a neural network model, Zipser and Andersen (1988) have shown how a population of such gain-modulated neurons could perform a transformation of eye-centered visual input into a head-centered reference frame, given the current eye position. This mechanism has later been formalized and 
extended to multi-directional transformations by Pouget and colleagues (Pouget and Sejnowski 1997; Denève et al. 2001).

A different line of experiments has found evidence for a predictive shift of retinocentric representations with every gaze change, consistent with the second hypothesis. Duhamel et al. (1992) described neurons in the lateral intraparietal cortex (LIP) that respond when a stimulus is about to be brought into their receptive field by a saccade, even if the stimulus is extinguished before the gaze actually changes. While this phenomenon has initially been described as a shift of receptive fields, Cavanagh et al. (2010) argued that it should more properly be interpreted as a remapping of attentional pointers. Such a retinocentric remapping could account for robust spatial memory without requiring a gaze-invariant representation, as has been shown in a neural model by Quaia et al. (1998). There is now substantial experimental evidence for remapping activity as a widespread characteristic of neural responses in both LIP (Kusunoki and Goldberg 2003; Heiser and Colby 2006) and FEF (Sommer and Wurtz 2006).

These two hypotheses are currently viewed as independent and potentially contradictory explanations for the phenomenon of trans-saccadic spatial memory (Colby and Goldberg 1999; Wurtz 2008). However, some previous modeling efforts have also hinted at possible connections between them. Specifically, it has been shown that gain-modulated neurons may also serve to produce a retinocentric remapping. In a neural network model of double-step saccades which relies on gain-modulated neurons, Xing and Andersen (2000) have observed that some of the neurons show activity patterns consistent with retinocentric remapping (see also White III and Snyder 2004; Cassanello and Ferrera 2007). The authors fail to account, however, for one of the most intriguing properties of the experimentally observed remapping activity, the fact that it can be observed well before the eye movement is completed. Moreover, remapping is restricted in this model to the representation of a single item that is selected as the target of a future saccade. Experiment, in contrasts, shows updating for stimuli that do not become targets of upcoming saccades (Duhamel et al. 1992).

In this paper, we will show how retinocentric remapping of multiple items in parallel can emerge from a bidirectional mechanism for reference frame transformation based on gain-modulated neurons. To account for the predictive nature of the remapping, we postulate that a similar mechanism predictively updates an internal representation of the current gaze direction. This mechanism uses a saccade signal that emulates a corollary discharge (CD) from the superior colliculus (SC). This is consistent with findings of Sommer and Wurtz (2004b, 2006), indicating that remapping activity in the FEF relies on such a CD signal. Other studies, in which eye position before a memory-guided saccade was perturbed, provided further support for the view that gaze direction is internally monitored primarily via CD signals, while propri- oceptive signals from the ocular muscles appear to play only a minor role (Guthrie et al. 1983; Bridgeman and Stark 1991).

Unlike most previous models of reference frame transformation, we assume that a single representation of gaze direction integrates eye and head position. This takes into account a growing amount of experimental data gathered from head-free animals which indicates that gaze shifts are planned and executed as a combination of eye and head movements (Freedman et al. 1996; Freedman and Sparks. 1997). Consistent with this, gain modulation by gaze direction as a combination of eye and head position has been observed in parietal cortex (Brotchie et al. 1995), resulting in body-centered maps (Snyder et al. 1998). We note that in humans and other primates, the changes of the actual gaze direction that lead to remapping events only occur at discrete times during the saccades, while eye and head positions may change in conjunction with each other before and after the gaze shift.

We use the theoretical framework of dynamic neural fields (DNFs) to formulate an architecture that accomplishes the predictive update of gaze direction, the formation and retention of gaze-invariant spatial memory, and saccadic remapping of retinocentric representations in a variety of stimulus settings. The neuronal architecture is broadly consistent with what is known about the underlying neural circuitry. The DNF framework enables us to model the change of activity patterns at a high temporal resolution. We account for an ensemble of experimental signature and provide a quantitative fit of neural remapping activity in the parietal cortex.

\section{Neurodynamic model}

\subsection{Dynamic neural fields}

DNFs are a class of neural models that describe the temporal evolution of population activities under the influence of external inputs and internal interactions (Wilson and Cowan 1973; Amari 1977), emphasizing the importance of attractor states and the transitions between them in the neural dynamics. Abstracting from the single spiking neurons that constitute a population (Eggert and van Hemmen 2001), DNFs use a continuous distribution of activation over an appropriate feature space to represent metric values (Fig. 1a). Feature dimensions used here include saccadic end-points, gaze angles, and the retinal positions of stimuli. The DNF model describes the evolution of activation distributions $a(x)$ over the feature space, which reflect the averaged membrane potentials in a neural population, through differential equations. The field equation (Amari 1977) takes the general form

$\tau \dot{a}(x)=-a(x)+h+i(x)+[f(a) * w](x)$,

with a time constant $\tau$, field resting level $h$, and an external input $i$. Lateral interactions within the field are implemented as a convolution of the field output, $f(a)$, with an interaction 
Fig. 1 Dynamic neural fields. A Activity distribution (thick gray line) in a DNF in response to an external stimulus (thin black line) under the influence of lateral interactions. B Lateral interaction kernels used in the model. Top Difference-of-Gaussians type kernel with local surround inhibition. Bottom Kernel with global inhibition.
A

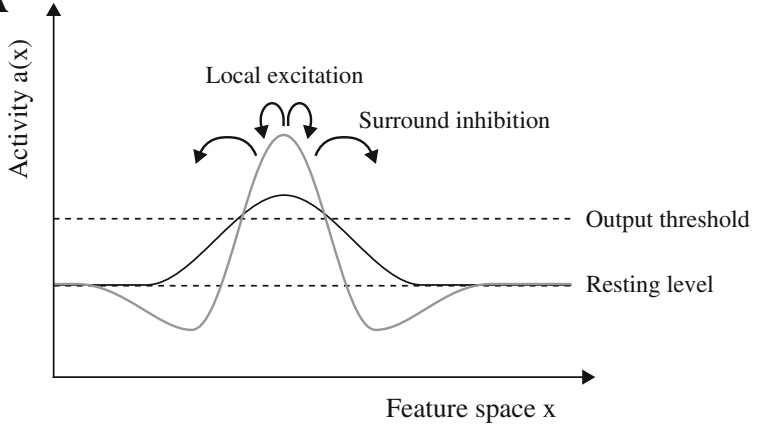

B

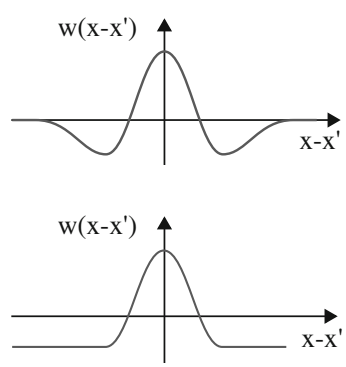

kernel $w$. This field output is computed for every field location as a sigmoid (logistic) function of the activation

$f(a)(x)=f(a(x))=\frac{1}{1+\exp (-\beta a(x))}$.

It reflects the average firing rate of a neurons in the population that support a certain feature value. It remains near zero for low activations, rises as activation reaches a soft threshold, and saturates at a value of one for high activations. All interactions between fields are likewise mediated by this same output.

Compared to more typical neural networks, the neural dynamics that govern DNFs are characterized by the capacity of neuronal interactions to take on a dominant role and generate stable states that may be sustained in the absence of input and may stabilize selection decisions. This is based on two typical forms of interaction within neural populationsmutual excitation between neurons responding to similar values, and mutual inhibition (via interneurons) between neurons coding for more dissimilar values. This pattern is reflected in the interaction kernels used here, which either take the form of a difference of Gaussians (with a "Mexican hat" shape) or a Gaussian shifted toward the negative by a constant (global) inhibitory component (Fig. 1b). These types of interactions promote the formation of localized peaks of activation in response to external stimulation (as shown in Fig. 1a).

Activation peaks are the units of representation in DNFs. Interaction kernels with global inhibition generate selection behavior in the field that promotes the formation of a single peak in response to multi-modal input. Difference-of-Gaussians interaction kernels enable the formation of multiple stable peaks that may represent the positions of multiple stimuli. If the lateral interactions have sufficient strength, activation peaks become self-sustained in the absence of external input, and provide a model of working memory (analogously to how working memory is modeled in networks of spiking neurons, e.g., Wang 2001). The interactions can readily be extended to multi-dimensional feature spaces. The DNF approach has successfully been employed in models of motor planning for both eye movements (Kopecz and Schöner 1995;
Trappenberg et al. 2001) and reach movements (Erlhagen and Schöner 2002; Cisek 2006). On a more abstract level, the approach is used in behavioral models of spatial cognition, visual working memory, and development (Thelen et al. 2001; Simmering et al. 2008; Johnson et al. 2008).

\subsection{Overview over the DNF architecture}

The model consists of several DNFs that are connected to each other by means of localized, excitatory projections, such that output in one field creates activation in a certain region of one or more other fields. Figure 2 shows the complete architecture of the model (simplified to one spatial dimension for ease of illustration). The six DNFs of this architecture can be divided into two largely independent modules: The saccade field (Fig. 2a), the two-dimensional (2D) update field (Fig. 2b), and the gaze field (Fig. 2c) form the gaze update module. In this module, the internal representation of the current gaze direction that is provided by the gaze field is predictively updated by a CD signal of an intended gaze change, represented in the saccade field. The second module, the transformation module, comprises the retinocentric field (Fig. 2d), the 2D transformation field (Fig. 2e), the bodycentered field (Fig. 2f), and the gaze field (Fig. 2c), with the latter acting as a common element of the two parts. This module is analogous to previous models of reference frame transformation (Zipser and Andersen 1988; Pouget and Sejnowski 1997), but it extends them to allow the parallel mapping of multiple locations from the retinocentric to the body-centered representation and back.

Next, we describe the architecture for reference frame transformation and retinocentric remapping in the transformation module (right part of Fig. 2), then the architecture of the gaze update module (left part of Fig. 2) which supports the transformation. The model represents visual space with its inherent two dimensions. For ease of illustration, we first discuss each module in a one-dimensional (1D) form and describe the extension to two dimensions thereafter. A full mathematical description of the $2 \mathrm{D}$ version is given in the appendix. 


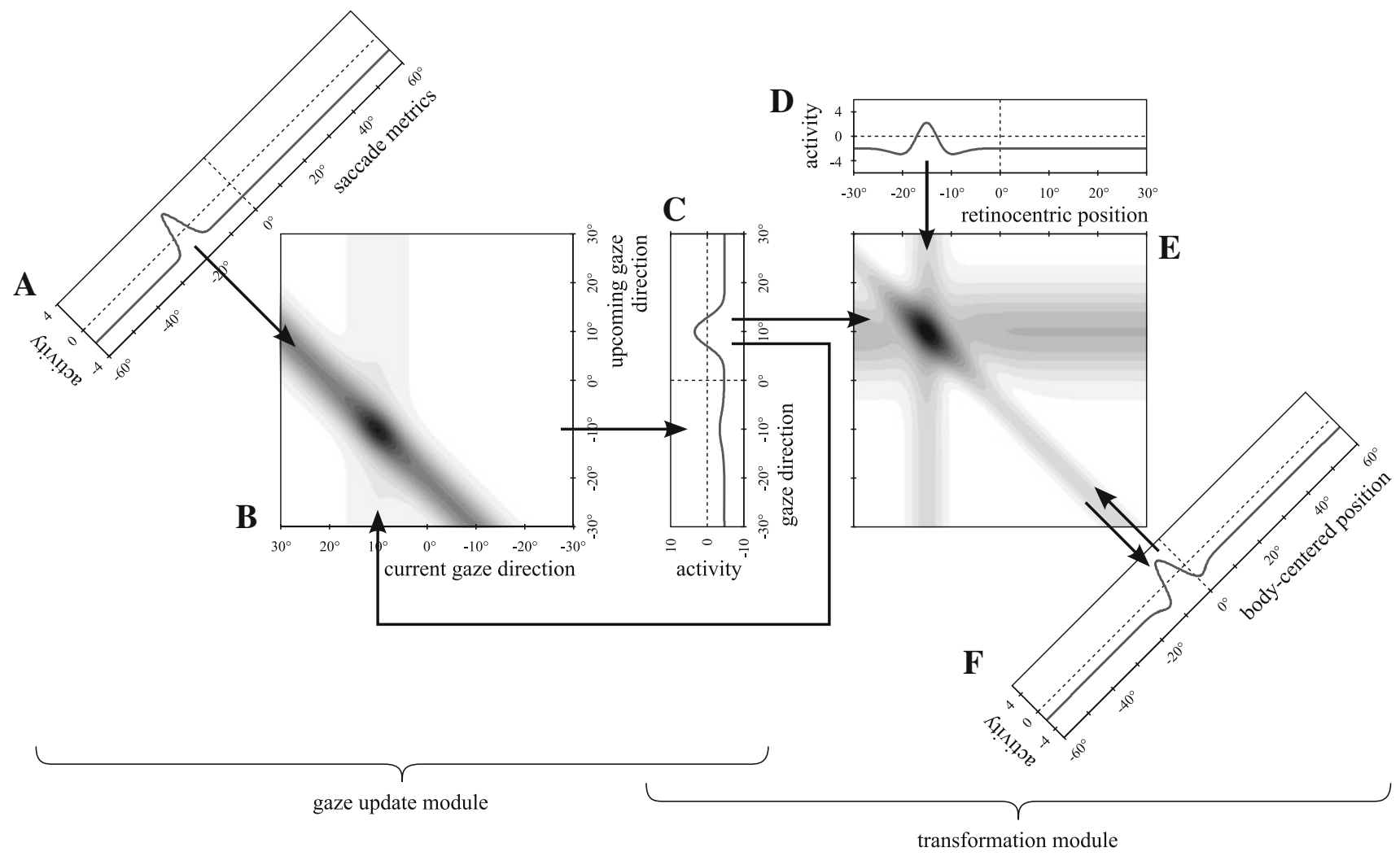

Fig. 2 Model architecture for the simplified case of 1D inputs. 1D fields $(\mathbf{A}, \mathbf{C}, \mathbf{D}, \mathbf{F})$ are shown as activity plots over their respective feature space, 2D fields $(\mathbf{B}, \mathbf{E})$ as activity distributions coded by brightness (darker meaning higher activity). Arrows between fields indicate local excitatory projections. The 1D fields are arranged such that these projections always run perpendicular to the feature space axis. Lateral interactions within fields are not shown. A Saccade field, B update field, $\mathbf{C}$ gaze field, $\mathbf{D}$ retinocentric field, $\mathbf{E}$ transformation field, $\mathbf{F}$ body-centered field. A detailed description of the architecture is given in Sect. 2

\subsection{Transformation module}

All visual information arrives through the retinocentric field. This field is defined over the space of retinocentric positions, covering a range of $-30^{\circ}$ to $30^{\circ}$, with the fovea at $0^{\circ}$ (see Fig. 2d; all position information in the model is given in angular coordinates). The dynamics of the retinocentric field, as well as all other 1D fields, follow Eq. 1. The field receives localized, excitatory external input, representing the locations of objects or salient regions. This input arrives after a fixed delay following the onset of a simulated visual stimulus, accounting for the transmission and processing time in the retina and early visual areas. It has a phasic-tonic temporal structure, with input strength being highest immediately after its onset and then decreasing to an intermediate level. Lateral interactions of the difference-of-Gaussians type are present in the retinocentric field, allowing several activity peaks to exist simultaneously (one at each location with sufficiently strong input). During a saccade, the visual input is turned off and the overall activity in this field is moderately suppressed, leading to the extinction of all activity peaks.

The current gaze direction is represented through the location of a single activity peak in the gaze field (Fig. 2c). This field covers the same range from $-30^{\circ}$ to $30^{\circ}$, with $0^{\circ}$ corresponding to the straight forward direction with respect to the body. There are strong lateral interactions in this field, consisting of local excitation and global inhibition. These interactions promote the presence of a single peak at all times, and they allow a peak to be sustained without external input once it is established.

The transformation field is defined over the combined space of retinocentric positions times possible gaze directions (Fig. 2e). It receives input from the retinocentric field and the gaze field along the corresponding axes: The input from the retinocentric field (shown on the horizontal axis in Fig. 2) activates the transformation field locally along the retinocentric dimension, and homogeneously over all gaze directions, forming vertical activity ridges. The gaze direction input (on the vertical axis) analogously creates horizontal ridges of activity, which are broader and stronger than those of the retinocentric input. This is described by a field equation of the form 


$$
\begin{aligned}
\tau \dot{a}_{\mathrm{T}}(x, y)= & -a_{\mathrm{T}}(x, y)+h_{\mathrm{T}}+\left[f\left(a_{\mathrm{T}}\right) * w_{\mathrm{TT}}\right](x, y) \\
& +\left[f\left(a_{\mathrm{R}}\right) * w_{\mathrm{TR}}\right](x)+\left[f\left(a_{\mathrm{G}}\right) * w_{\mathrm{TG}}\right](y) \\
& +\left[f\left(a_{\mathrm{B}}\right) * w_{\mathrm{TB}}\right](x+y),
\end{aligned}
$$

with $a_{\mathrm{T}}$ being the transformation field, $a_{\mathrm{R}}$ the retinocentric field, $a_{\mathrm{G}}$ the gaze field, and $a_{\mathrm{B}}$ the body-centered field (whose function and connectivity is described below). The lateral interaction kernel $w_{\mathrm{TT}}$ takes the form of a $2 \mathrm{D}$ difference of Gaussians, the interaction kernels $w_{\mathrm{TR}}, w_{\mathrm{TG}}$, and $w_{\mathrm{TB}}$ are all Gaussians that smooth the field outputs before feeding them as inputs to the transformation field. The biological interpretation of the activation distribution described by this field is discussed in Sect. 3.3.

The two inputs from the retinocentric and the gaze field are combined additively in the transformation field, but through the output non-linearity and the lateral interactions in the field, it effectively performs an "and" operation on the inputs: While each of the input ridges for itself is not sufficient to produce significant output, activity peaks form at the intersection point between two ridges. If multiple items are represented in the retinocentric field, each of them will produce a peak in the transformation field, lined up along the single activity ridge induced by the gaze field.

The combined information in the transformation field is sufficient to determine the body-centered position of each visual stimulus: The position $\mathbf{p}_{\mathrm{B}}$ of an item in the body-centered reference frame is given by the sum of its retinocentric position $\mathbf{p}_{\mathrm{R}}$ and the gaze direction vector $\mathbf{v}_{\mathrm{G}}$ (all in angular coordinates), $\mathbf{p}_{\mathrm{B}}=\mathbf{p}_{\mathrm{R}}+\mathbf{v}_{\mathrm{G}}$. It follows from this relationship that all combinations of retinocentric position and gaze direction that correspond to the same body-centered position lie on a diagonal line through the transformation field. We therefore sum up all field output along the diagonal and project it onto the body-centered field (Fig. 2f). The body-centered field features the same lateral interactions as the retinocentric field, but it covers twice the range $\left(-60^{\circ}\right.$ to $\left.60^{\circ}\right)$, such that all possible combinations of retinocentric position and gaze direction lead to a valid position in this field. The body-centered field projects back to the transformation field along the same path as it receives input, leading to diagonal activity ridges in the transformation field (Fig. 2e ; Eq. 3). Activity peaks in the transformation and body-centered field are thus coupled through mutual excitation.

The system can be operated in two different modes, which can be equated with different attentional states that depend on the current task: In the memory mode, the coupled peaks in the transformation and the body-centered field are self-sustained, due to the lateral interactions and mutual excitation between the fields. Once peaks have formed, representing the locations of visual stimuli, they persist even after these external stimuli are switched off and the input from the retinocentric field ceases. In the perceptual mode, the peaks that form in response to external input decay after a short time if the input is turned off. The two modes differ only in the baseline activity level of the body-centered field. The higher baseline activities in the memory mode can be interpreted as the result of a global modulatory input. In the simulations, we manually set the appropriate mode depending on whether the task that is modeled requires subjects to memorize stimulus locations or not.

The extension of the transformation module to $2 \mathrm{D}$ visual information and gaze direction is straightforward. The architecture and the connection patterns for this case are shown in Fig. 3. The retinocentric, gaze direction, and body-centered fields here span two dimensions each, with their respective lateral interactions extended accordingly (Fig. 3a). The transformation field, which was 2D in the simpler scenario, now describes an activity distribution over four dimensions (two for retinocentric position, two for gaze direction). In analogy to the ridges in the $2 \mathrm{D}$ transformation field, the retinocentric and gaze direction inputs activate regions in the four-dimensional (4D) field with the same preferred retinocentric position or gaze direction, respectively (Fig. 3b, c). Each position in the body-centered field interacts bidirectionally with all positions in the transformation field that show a combination of preferred retinal location and gaze direction which matches the preferred body-centered location (Fig. 3d). Lateral interactions in the transformation field are consistently extended in four dimensions, such that regions affected by lateral excitation and inhibition from one point form ellipsoids in 4D space (Fig. 3e). A similar formulation (without lateral interactions in the high-dimensional representation) has been used in a model of object recognition by Fazl et al. (2009).

\subsection{Gaze update module}

The second part of the mechanism is concerned with the update of the internal representation of gaze direction by a CD signaling a gaze shift. Like the reference frame transformation, this update can be described by a vector addition, $\mathbf{v}_{\mathrm{G}}^{\text {new }}=\mathbf{v}_{\mathrm{G}}^{\text {old }}+\mathbf{v}_{\mathrm{S}}$, with $\mathbf{v}_{\mathrm{S}}$ being the saccade vector that describes the direction and size of the gaze change (in retinocentric coordinates). Therefore, a mechanism similar to the reference frame transformation can be used here, with the difference that the output of the computation is fed back into one of the input fields (see left part of Fig. 2). Again, we will first describe the architecture for 1D inputs.

The saccade field (Fig. 2a) represents the metrics of an upcoming saccade in a retinocentric reference frame, covering a range from $-60^{\circ}$ to $60^{\circ}$ (such that transitions from one end of the gaze field to the other can be modeled). It only passively conveys information corresponding to a $\mathrm{CD}$ signal, showing a single, input-driven peak of fixed duration for each saccade. We do not explicitly model the processes of saccade target selection and saccade initiation, although the 
Fig. 3 Architecture and connection patterns of the remapping mechanism for $2 \mathrm{D}$ inputs. A Architecture overview. The 4D transformation field is visualized in a $2 \mathrm{D}$ plot subdivided into tiles. The units within each tile share the same preferred gaze direction and span all possible retinocentric positions. Activities in all fields are color coded (red meaning highest, blue lowest activity). B Connection pattern of retinocentric input to the transformation field (in a low-resolution implementation of the architecture). The input strength from one activated position in the retinocentric field is color coded in the transformation field.

C Connection pattern of gaze-direction field input. D Connection pattern of body-centered field input. E Pattern of lateral connections in the transformation field (excitatory and inhibitory, the latter visible as darker blue areas), originating from the central position in the central tile. (Color figure online)

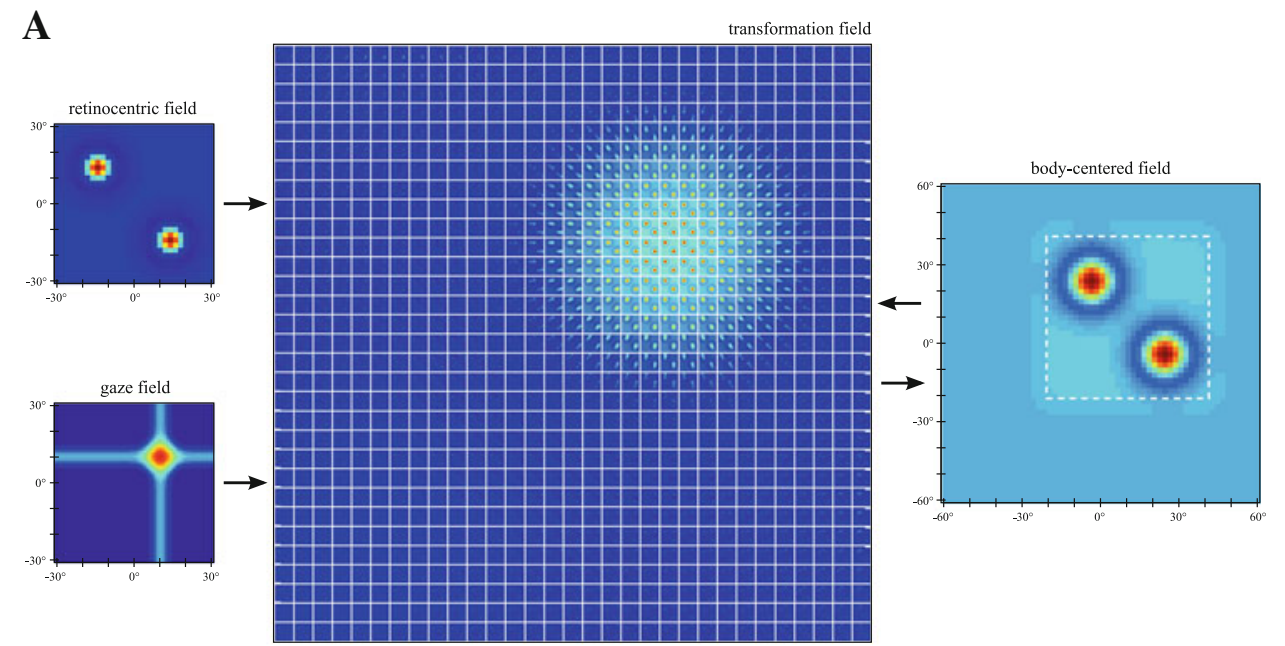

B

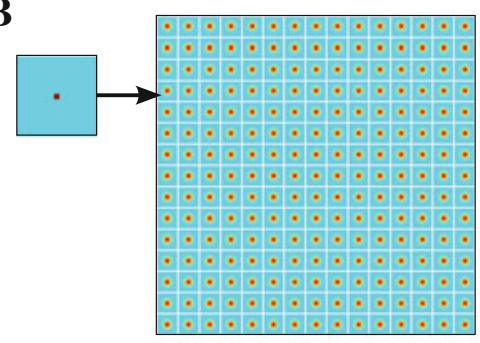

D

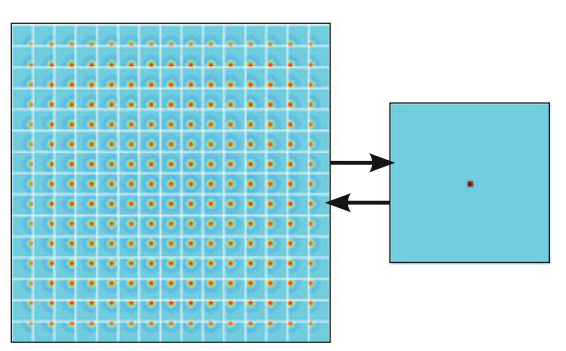

C

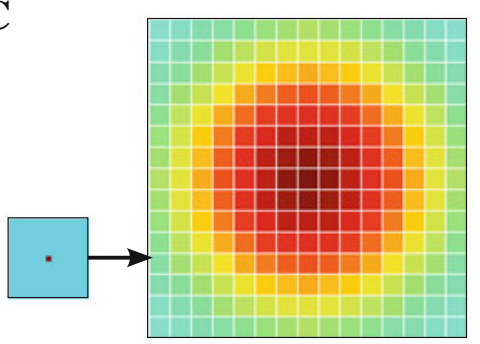

$\mathbf{E}$

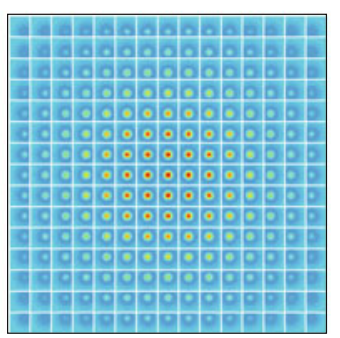

retinocentric field or the transformation field (when read out along the retinocentric dimension) could provide the input for this. In our previous work, we have provided detailed models of saccade planning that are compatible with our current architecture (Kopecz and Schöner 1995; Wilimzig et al. 2006; see also Quaia et al. 1999; Trappenberg et al. 2001). Here, we only represent the result of this process by applying an input to the appropriate location in the saccade field for every gaze shift required in the simulations. The input has a constant strength and a duration of $100 \mathrm{~ms}$. The actual gaze shift is assumed to start $50 \mathrm{~ms}$ after the onset of this signal and take $50 \mathrm{~ms}$ to complete. During this latter interval, the visual input is turned off and the activity in the retinocentric field is globally suppressed. The saccade signal that we use emulates in simplified form the perisaccadic activity that has been described for SC, thalamus, and FEF by Sommer and Wurtz (2004a), and that is conjectured to be a CD signal conveying saccade metrics to the cortex.
The update field is defined over the 2D space spanned by the current and the upcoming gaze direction (Fig. 2b). The current gaze direction represented in the gaze field (Fig. 2c) is fed into the update field along one dimension (Fig. 2, arrow that circles around the update field), leading to a homogeneous vertical activity ridge. The saccade field projects diagonally into the update field and can provide a stronger input than the gaze field. This yields the field equation

$$
\begin{aligned}
\tau \dot{a}_{\mathrm{U}}(x, y)= & -a_{\mathrm{U}}(x, y)+h_{\mathrm{U}}+\left[f\left(a_{\mathrm{U}}\right) * w_{\mathrm{UU}}\right](x, y) \\
& +\left[f\left(a_{\mathrm{G}}\right) * w_{\mathrm{UG}}\right](-x)+\left[f\left(a_{\mathrm{S}}\right) * w_{\mathrm{US}}\right] \\
& (x+y),
\end{aligned}
$$

with $a_{\mathrm{U}}$ being the update field and $a_{\mathrm{S}}$ the saccade field, $w_{\mathrm{UU}}$ the lateral interaction kernel (with a Gaussian excitatory and a global inhibitory component), and $w_{\mathrm{UG}}$ and $w_{\mathrm{US}}$ Gaussian interaction kernels between the fields.

While the constant gaze direction ridge alone does not evoke significant output in the update field, the two input 
ridges together are sufficient to produce a localized peak at their intersection point (illustrated in Fig. 2b). Unlike in the gaze field, peaks in the update field are not self-sustained: While they will remain stable without the gaze input, they decay as soon as the saccade signal ceases. The update field projects back into the gaze field through a horizontal projection. Incoming and outgoing projections of the gaze field thus run perpendicular to each other in the update field.

To extend this architecture to $2 \mathrm{D}$ gaze direction, we combine two 1D gaze update modules, one for horizontal, and one for vertical gaze direction. Unlike for the reference frame transformation, no correspondence problem can occur here as there is only a single gaze direction at all times. We merge the two 1D saccade fields into a single 2D field, which is read out along the two axes to obtain the respective inputs to the update fields. The update and gaze-direction fields for both dimensions remain completely separated. An additional 2D gaze field is added to combine gaze information from the horizontal and the vertical field. The $2 \mathrm{D}$ gaze field holds a single peak at all times, driven by inputs from the two 1D fields along the respective dimensions and softly normalized through global inhibitory interactions. This 2D gaze field then provides the input to the transformation field for the $2 \mathrm{D}$ case.

\section{Simulation results}

\subsection{Update of gaze direction}

The gaze update mechanism we propose computes the new gaze direction by adding the metrics of the intended saccade to the current gaze direction. The addition process consists of a fixed sequence of transitions between different stabilized states (Fig. 4): Between gaze shifts, a single activity peak is present in the gaze field, which is kept stable without any external input by means of the lateral interactions (Fig. 4a). The gaze field projects a ridge input into the update field, but activity here remains clearly below the output threshold.

When a saccade signal arrives (forming a localized activity peak in the saccade field), a stronger diagonal ridge is projected into the update field, and a self-stabilized peak emerges at the intersection point of the two input ridges (Fig. 4b). The geometry of the fields and the projections between them ensures that the vertical position of this peak is $v_{\mathrm{G}}^{\text {new }}=v_{\mathrm{G}}^{\text {old }}+v_{\mathrm{S}}$. This peak in turn projects horizontally to the same position $v_{\mathrm{G}}^{\text {new }}$ in the gaze field. A strong activity peak is created at this new location, and it suppresses the previous peak by means of global inhibition. After a brief transition period during which two competing peaks are present in the gaze field, the new peak prevails, being again self-stabilized and independent of the external input (Fig. 4c).
Importantly, the peak in the update field remains stable during this time (Fig. 4c): Due to its strong self-excitation, it does not collapse or shift to a new location when the original input from the gaze field ceases. The global inhibition from this peak also prevents the formation of another peak at the intersection of the still active saccade input and the input coming from the new gaze direction peak. Without this suppression, a single saccade signal would potentially produce a cascade of several gaze updates. With the lateral interactions in place, however, the single peak in the update field only decays after the saccade signal has ceased, at which time the system returns to its initial state (Fig. 4d).

We tested this mechanism by producing a peak at different positions in the saccade field (with timing as described in Sect. 2.4), simulating gaze shifts from $0^{\circ}$ to $40^{\circ}$ in steps of $1^{\circ}$ both horizontally and vertically (in all combinations). The initial gaze direction for all trials was set to $\left(-20^{\circ},-20^{\circ}\right)$, such that the expected gaze direction after the saccade was within the range of $\left[-30^{\circ}, 30^{\circ}\right]$ covered by the gaze field. The resulting gaze direction was determined as the center of mass of the gaze field output. For all tested saccade metrics, the activity for the new gaze direction started to rise around or briefly after the beginning of the saccade. By the time the saccade ended, the update was always completed. At this point, the mean error (deviation of the represented from the expected gaze direction) was $0.08^{\circ}$ (maximal error: $0.53^{\circ}$, standard deviation from the expected gaze direction: $0.14^{\circ}$ ).

This gaze update mechanism fulfills two critical requirements to be used as the basis of a predictive remapping: First, it only uses information that is available before the actual gaze shift is initiated. The signal driving the update of the gaze representation is a space-coded representation of the saccade metrics, as it is found in the SC, the thalamus and the FEF briefly before a saccade (Sommer and Wurtz 2004a). Second, the mechanism itself performs a fast direct computation of the new gaze direction, thereby making the result of the gaze update available before the motor act is completed. This separates it from the mechanisms that are used in many models of the ocular motor system (e.g., Goossens and Van Opstal 2006), which rely on an integration of a motor signal over time (and which we still assume to exist in addition to the system presented here).

In addition to these properties, the type of computation we employ is very robust against fluctuations in the timing of the saccade signal. Varying the saccade signal duration within a range of $\pm 25 \%$ of its default value only leads to a moderate increase in the maximal error $\left(0.86^{\circ}\right)$ and virtually no change in the mean error $\left(0.08^{\circ}\right.$, standard deviation from expected gaze direction: $0.15^{\circ}$ ). While we assume that proprioceptive signals are not critical for the internal monitoring of gaze direction, the architecture is still open to incorporate such slower signals as additional inputs to the gaze field, which may stabilize its activation profile and keep it calibrated over 

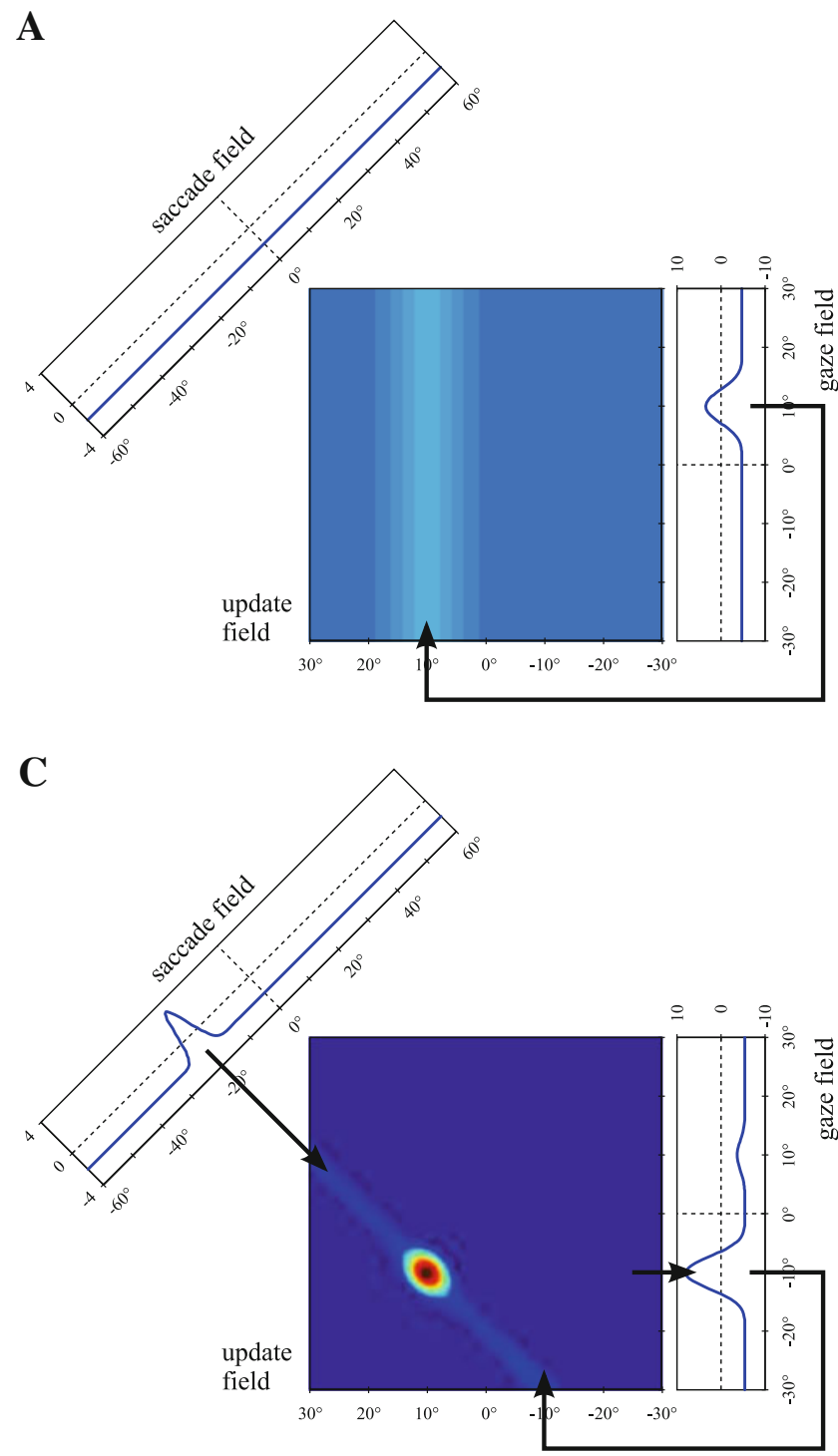

Fig. 4 Evolution of field activities during an update of gaze direction. Fields are shown as in Fig. 2 (left part only), activities in the update field are color coded (red meaning highest, blue lowest activity). Arrows between fields indicate dominant directions of information flow during each time period, the underlying connectivity remains fixed. A Activity distribution between saccades, with a peak in the gaze field projecting sub-threshold activity into the update field. B A peak in the saccade field indicates a $20^{\circ}$ leftwards saccade. It projects into the update field,

time. DNFs are well suited to integrate different sources of information into a robust estimate of a metric value like the current gaze direction (see e.g., Erlhagen and Schöner 2002).

The patterns of activation in the update field show an intriguing analogy to neural responses in the central thalamus described by Tanaka (2007). Neurons in this area are assumed to convey eye position information to the cortex. The representation of eye position is decomposed into a horizontal and a vertical component at this stage, as is also the case in our
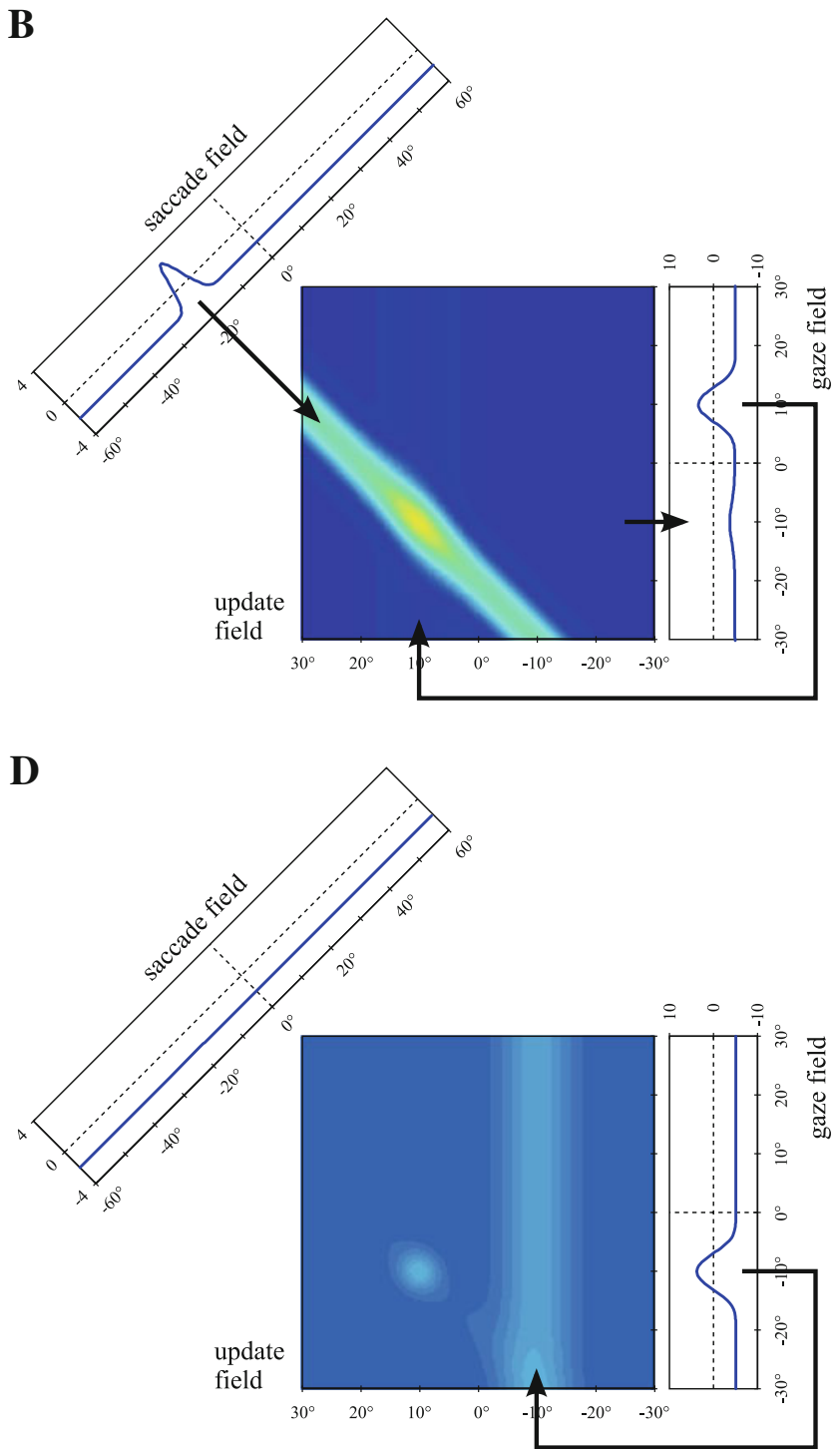

intersecting the gaze field input and forming a peak that projects back into the gaze field. C The peak in the update field is stabilized by strong local excitatory and global inhibitory interactions. Its input to the gaze field has formed a new activity peak there, which suppresses the previous peak. D After the saccade signal has ceased, the peak in the update field collapses and the system returns to the state shown in (a), with the gaze direction peak shifted by $20^{\circ}$ to the left. (Color figure online)

model. Many of the neurons in Tanaka's study changed their firing rate before the eye position itself had changed. Most interestingly, a subpopulation of neurons showed a significant modulation of their response by the metrics of the saccade that led or was about to lead to a given eye position. This resembles the activation profile of units in the update field of our model, whose response likewise depends on both the current gaze direction and the metrics of the current saccade. Tanaka considered this response modulation an undesirable 
bias in the representation of eye position. We suggest that it may be the signature of an update mechanism like the one we have described.

\subsection{Emergence of retinocentric remapping from reference frame transformations}

An inherent property of our architecture is a shift of activity peaks with respect to the retinocentric reference frame during every gaze change. We will explicate this in the simplified version for 1D inputs with a single perceptual item (the mechanism is the same for $2 \mathrm{D}$ inputs). The evolution of field activities in the transformation module for this case is shown in Fig. 5. In order to illustrate the retinocentric shift more clearly, the figure also shows the projection of the transformation field output onto the retinocentric dimension (determined by summing up the output over the dimension of gaze direction, see red plots in Fig. 5). A projection of this kind allows it to read out the current retinocentric positions of all memorized items, and may be used in saccade planning or attentional modulation of earlier visual areas. Note that we do not feed this output back into the retinocentric field, as we consider it distinct from the driving visual input represented in this field.

When an external stimulus is applied, it first forms an activity peak in the retinocentric field. Activity is then propagated into the transformation field, and the retinocentric position information is combined with the gaze information to perform the forward transformation into the body-centered frame of reference (Fig. 5a). This results in the formation of a distributed representation of the stimulus position in two different reference frames (Fig. 5b). The two peaks in the transformation field and the body-centered field are stabilized through lateral interactions within their respective field and through mutual excitation along the diagonal axis. The activity in the retinocentric field is always suppressed at the beginning of a saccade, such that only these two coupled peaks remain.

The saccade signal is processed in the gaze update mechanism, which leads to the emergence of a new peak in the gaze field. This creates another input ridge to the transformation field, which intersects the diagonal ridge from the body-centered field (Fig. 5c). At this intersection point, a new peak will form, which is offset from the old one along both the dimension of gaze direction and retinocentric position. At the same time, the old input ridge from the gaze field decays, and the peak that was situated on it vanishes without this input (Fig. 5d). In effect, the activity peak in the transformation field jumps to another position along the diagonal activity ridge, driven by the change of the gaze direction input. This also produces a shift of the activity peak's position with respect to the retinocentric reference frame: It is shifted by the inverse of the saccade vector, and thus moved to the new retinocentric position of the stimulus that originally produced the activity peak (assuming that the stimulus position is fixed in the world). This can be seen most clearly in the retinocentric output of the transformation field (red plots in Fig. 5).

It is noteworthy that the position of the peak in retinocentric coordinates jumps from one location to anotherthat is, the activity at the old position decays, and at the same time rises at the new position, without a significant increase of activity in between the two locations (this is true for sufficiently large saccades, for which the active regions before and after the gaze change do not overlap; otherwise the response pattern is more akin to a smooth drift of the activity peak). This pattern in the model matches observations of Sommer and Wurtz (2006) for remapping activity in FEF. It is also consistent with experimental evidence that the locus of attentional facilitation does not slide over intermediate locations during a gaze change (Golomb et al. 2011). In contrast, those data are in conflict with the neural network model of Keith et al. (2010), in which a saccade signal present both before and during the gaze change generally produces remapping activity that propagates continuously from the old to the new retinocentric location. In this respect, that model can be refuted.

To fully account for experimental results, it is critical that the remapping can be applied to multiple items simultaneously. As all brain areas in which remapping has been observed (LIP, FEF, and SC) are also involved in the planning of eye movements, we have to assume that the saccade target is generally saliently represented in these areas. Many experiments related to the remapping hypothesis (including the setup by Duhamel et al. 1992) focus on additional items in the scene that are not the target of the current saccade. Figure 6 shows the evolution of activities in the model for a case in which two visual stimuli are present, a saccade is made to foveate the outer one and both locations are remapped. The locations of multiple items are represented in the model through the presence of multiple peaks in the retinocentric, transformation, and the body-centered fields (Fig. 6a). The peaks in the transformation field are lined up on the input ridge from the gaze field, while the diagonal input ridges from the body-centered field run parallel to each other. When the gaze-direction input changes, all peaks in the transformation field will shift in parallel along their respective diagonal ridge (Fig. 6b). This way, the new retinocentric position of each item is predicted without interference between them (see red plot in Fig. 6b). Figure 6a shows that in the case of multiple visual inputs, additional intersections of input ridges occur in the transformation field (between the input from one retinocentric peak and the input from a non-corresponding body-centered peak). No self-stabilized peaks will form at these intersection points, because the presence of the stronger gaze input ridge is a prerequisite to reach the transformation field's output threshold. 
A

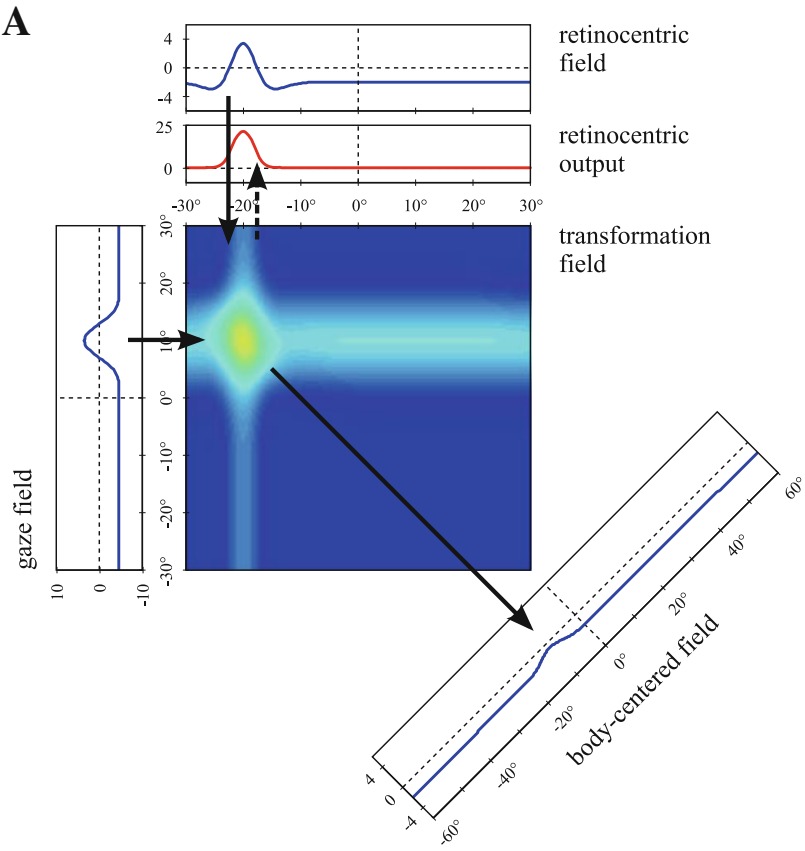

C

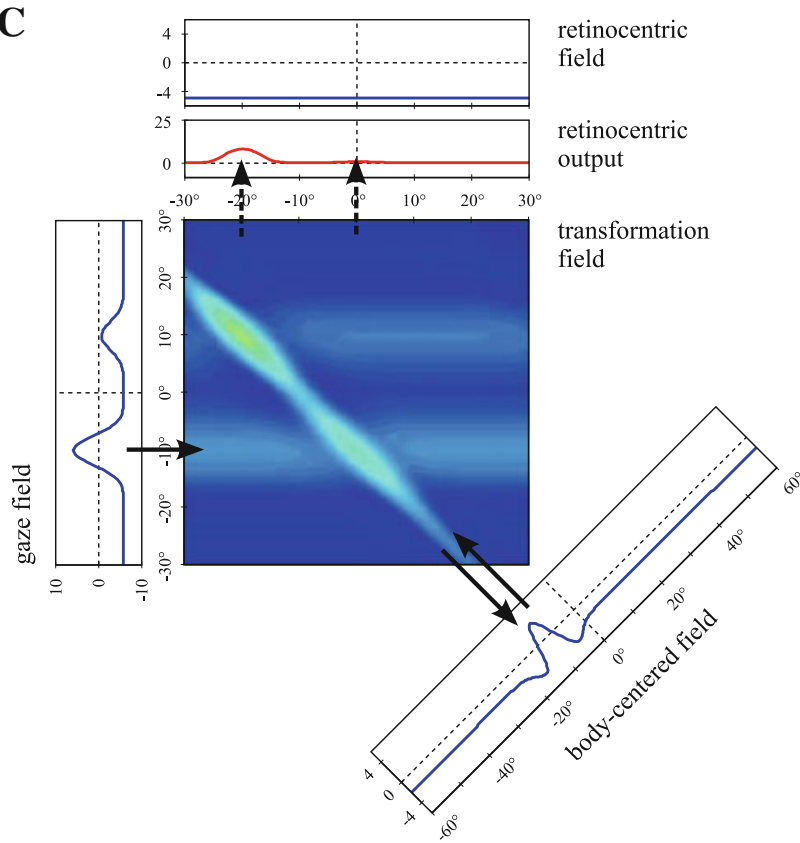

Fig. 5 Evolution of field activities for a retinocentric remapping. Fields are shown as in Fig. 2 (right part only). The red plot below the retinocentric field additionally shows the transformation field output, projected back into the retinocentric reference frame. A Situation briefly after visual stimulus onset, with peaks in the retinocentric and the transformation field and a peak about to form in the body-centered field. B After the visual stimulus is turned off, the activity peaks in the transformation and the body-centered field yield a distributed and coupled

\subsection{Gain modulation in the transformation field}

In order to show that the processing in the model is consistent with neural responses in the parietal cortex, we will com-

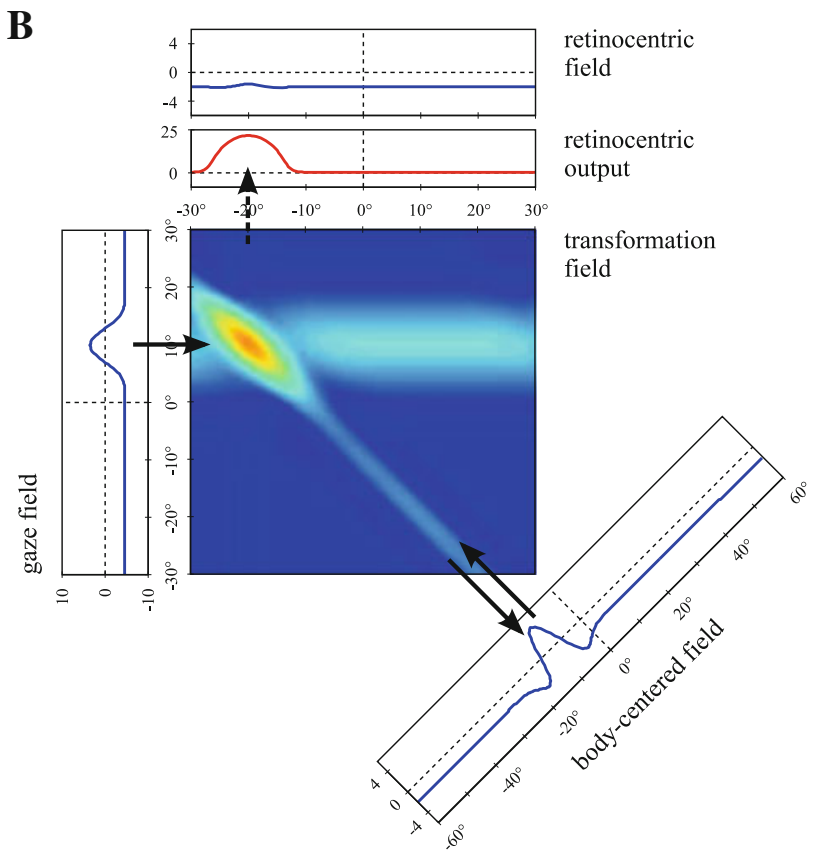

D

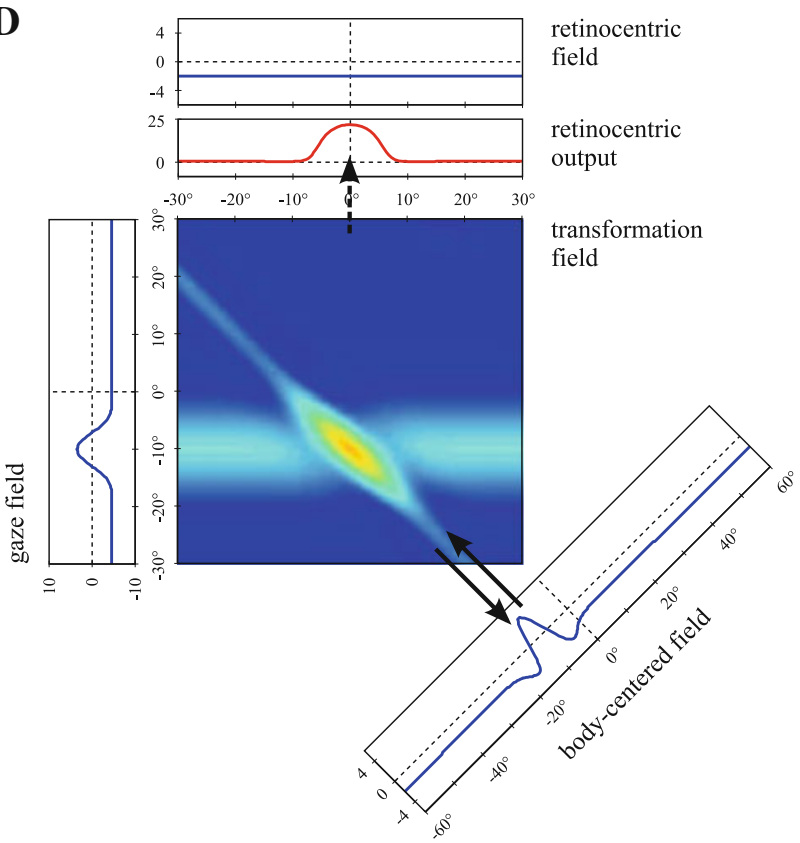

memory representation of its location. $\mathbf{C}$ At the time of a gaze change, a new peak forms in the gaze field, producing a new input ridge in the transformation field. While the original peak in the transformation field quickly decays, a new peak forms at the intersection between the new gaze input and the persisting input from the body-centered field. D At the end of the gaze change, the peak in the transformation field has shifted to a new location, driven by the changing gaze direction input. The body-centered representation remains unchanged. (Color figure online)

pare the transformation field output with the firing patterns of gain-modulated neurons, as they were identified in the parietal cortex by Andersen and colleagues (Andersen et al. 1985; Brotchie et al. 1995). These neurons are visually responsive 


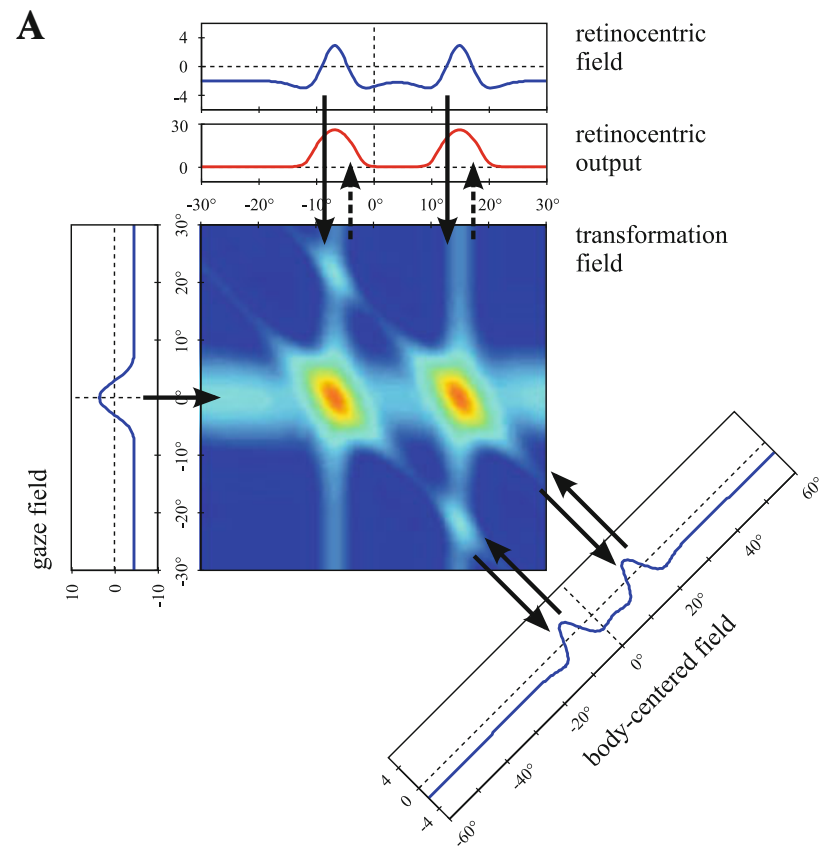

Fig. 6 Evolution of field activities for the parallel remapping of two items. Fields are visualized as in Fig. 5. A The two item locations are mapped in parallel from the retinocentric to the body-centered reference

and fire if a stimulus is presented at a certain (often relatively broad) retinal location. When the measurements are repeated under different gaze directions (using different fixation points on a screen), the retinocentric receptive fields of these neurons remain largely fixed, but the strength of their response to visual stimulation as well as their firing rate in the absence of stimulation change significantly (Andersen et al. 1985). Key response properties of gain-modulated neurons are reproduced in the transformation field.

Figure 7 shows the output at a single position in the transformation field for different stimulus positions and gaze directions (obtained from the full implementation for 2D inputs, from which all the following results are generated). Figure 7a shows the field output briefly after stimulus onset. The output becomes maximal for a fixed position of the visual stimulus, independent of the current gaze direction. The strength of the response, however, is strongly modulated by the current gaze direction, and the response disappears if the gaze deviates too far from a certain preferred value. In addition, the gaze direction also changes the base output in the absence of visual stimuli near the preferred location.

Later during the stimulus presentation, the output pattern in the transformation field undergoes some changes (Fig. 7b): When the body-centered representation has formed and provides an additional input to the transformation field, the preferred retinocentric position shows a partial shift as the gaze

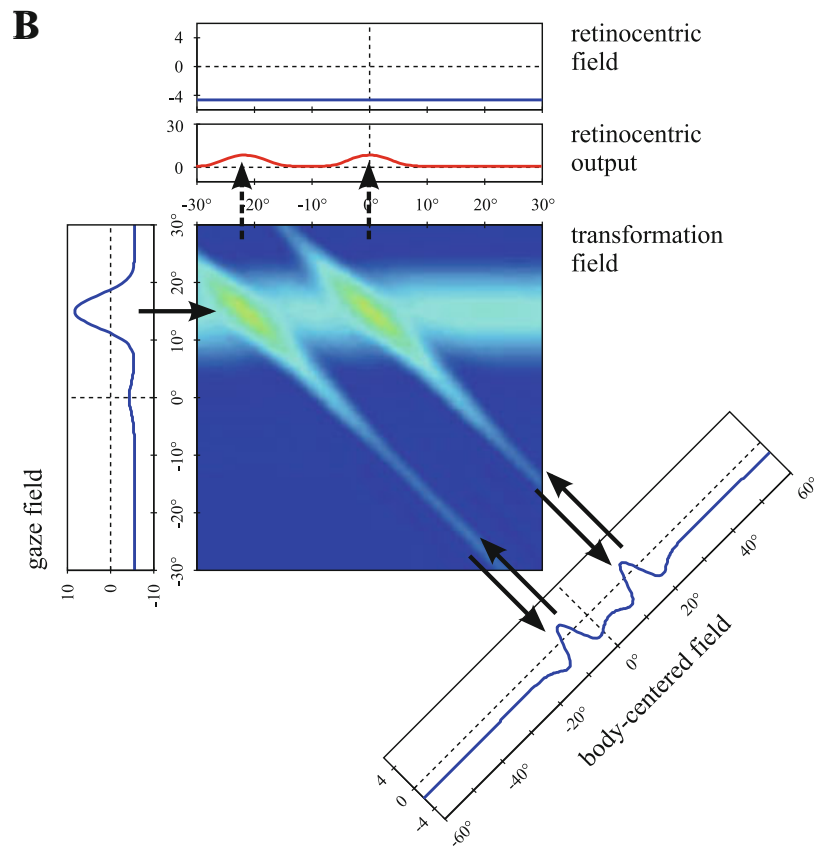

frame. B The two peaks that have formed in the transformation field simultaneously shift their position as the gaze direction input changes. (Color figure online)

direction changes (compare also Fig. 6a, b). Such a response pattern can be interpreted as representing a location in a reference frame that is intermediate between retinocentric and body-centered. Comparable partial shifts of receptive fields under changing gaze directions have been observed in bimodal neurons (showing visual and auditory or tactile responses) in LIP and the ventral intraparietal area (Stricanne et al. 1996; Avillac et al. 2005). This effect has been interpreted as the result of converging inputs from different reference frames, which is consistent with the mechanism described here.

The modulation of the visual response by the gaze direction in the model is a result of the summation of visual and gaze inputs, a sigmoidal output function, and lateral interactions in the field. As shown in an earlier model by Salinas and Abbott (1996), such modulation does not require multiplication of gaze and visual stimulus inputs. The gain modulation within the update field is based on broad localized gain fields. Each point in the transformation field has one preferred gaze direction, and its response strength decreases for any deviation from this direction. This type of modulation generates spatial representations that consist of localized, self-stabilizing activity peaks. A similar mechanism has been used by Denève et al. (2001), while many previous descriptions and models of gain-modulated neurons had assumed linear or planar modulation based on a linear eye position signal 

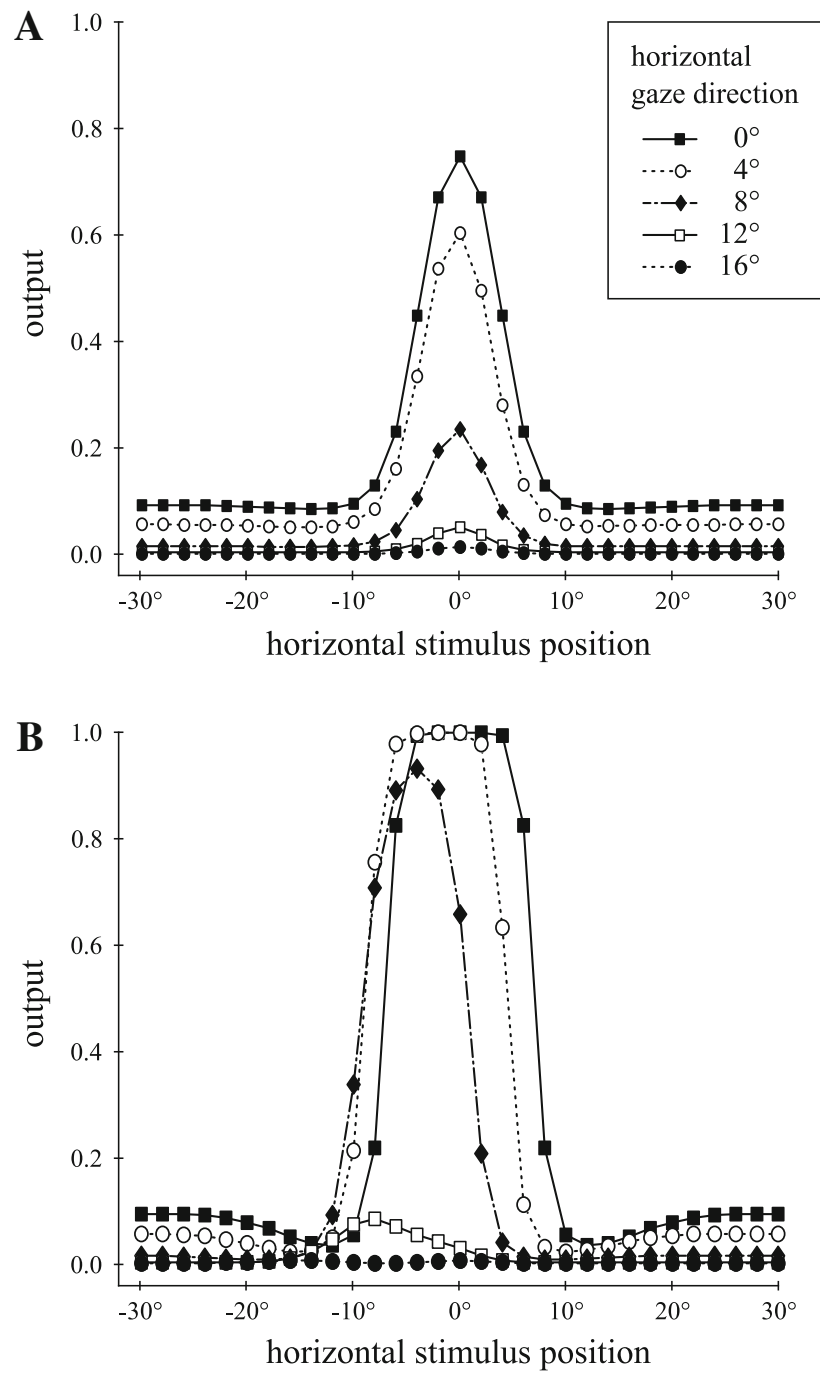

Fig. 7 Modulation of visual response by gaze direction in the transformation field. The plots show the output at the central position in the transformation field (with respect to both the retinocentric and the gaze dimensions) in response to a visual stimulus presented at different horizontal locations and for different horizontal gaze directions (vertical stimulus location and vertical gaze direction was always $0^{\circ}$ ). To increase the clarity of the plot, only responses for positive gaze directions are shown. Responses for negative gaze directions are symmetrical to the ones depicted. A Output $75 \mathrm{~ms}$ after stimulus onset. At this time, a peak has formed in the transformation field, but not in the body-centered field. The local response in the transformation field shows a fixed and approximately symmetrical visual receptive field around $0^{\circ}$ and a strong modulation by gaze direction, which is also symmetrical with respect to the preferred gaze direction of $0^{\circ}$. B Output $150 \mathrm{~ms}$ after stimulus onset. At this time, the distributed representation with peaks in the transformation and the body-centered fields has formed. The response now shows saturation effects (due to the sigmoid output function), more pronounced lateral inhibition and a partial shift of the visual receptive field with changing gaze direction.

(Pouget and Sejnowski 1997; Xing and Andersen 2000). We note that both localized and planar as well as mixed gain fields have been observed in experimental studies for LIP (Andersen et al. 1985). Likewise, neurons sensitive for eye position were found in area V6a with both localized and monotonic response fields (Nakamura et al. 1999 ${ }^{1}$ ). The authors of this study draw attention to the problem of classifying neurons that respond most strongly for a position at the edge of the grid of tested eye position (which is often quite limited). The firing rate of such neurons can often be fitted well with a monotonic response behavior, although they may, in fact, posses a localized response field that extends beyond the tested area. This problem may lead to an overestimation of the number of neurons with planar gain fields in LIP.

Do neurons exist that are tuned within a body-centered reference frame? In the model, the body-centered field serves primarily to mediate the diagonal projections from and to the transformation field, which are the basis for the remapping. The body-centered field does not by itself provide an independent spatial representation. For instance, without the contribution from the transformation field, it does not support self-sustained peaks. We have tested whether the body-centered field can be replaced by direct connections within the transformation field along the diagonals. Within the model at its current settings, we achieved satisfactory results only under limited conditions, and that only if we operated in single-peak mode. The explicit body-centered field greatly increases the stability of the mechanism and, in particular, eliminates the risk of an explosion of activity in the transformation field. So the questions are: Is the bodycentered population actually needed and does it exist in the brain? To this date, no explicit body-centered representation has been identified in the posterior parietal cortex. A subset of bimodal (visual and tactile) neurons has been found, however, that respond in a gaze-independent (head-centered) reference frame even when stimulated visually (Avillac et al. 2005). At this point, both questions must remain somewhat open. What is critical for the model is that the body-centered information is implicitly available by projection out of the transformation field along the diagonal.

\subsection{Trans-saccadic spatial memory}

To test whether the remapping mechanism detailed above can account for robust spatial memory in the face of gaze changes, we simulated a sequential saccade tasks. We used the full implementation of the model with $2 \mathrm{D}$ visual input, and operated the system in the memory mode. The simulated task is illustrated in Fig. 8. First, two visual stimuli are presented consecutively for $50 \mathrm{~ms}$ each (Fig. 8a, b). We set $t=0 \mathrm{~ms}$ to be the time of the first stimulus onset, the onset of the second stimulus is then at $t=200 \mathrm{~ms}$. A saccade signal indicating a gaze change to the first stimulus is given

\footnotetext{
1 The neurons observed in this study only change their firing rate after a gaze change has been completed, and can therefore not be equated directly to the gaze field in the model.
} 
A
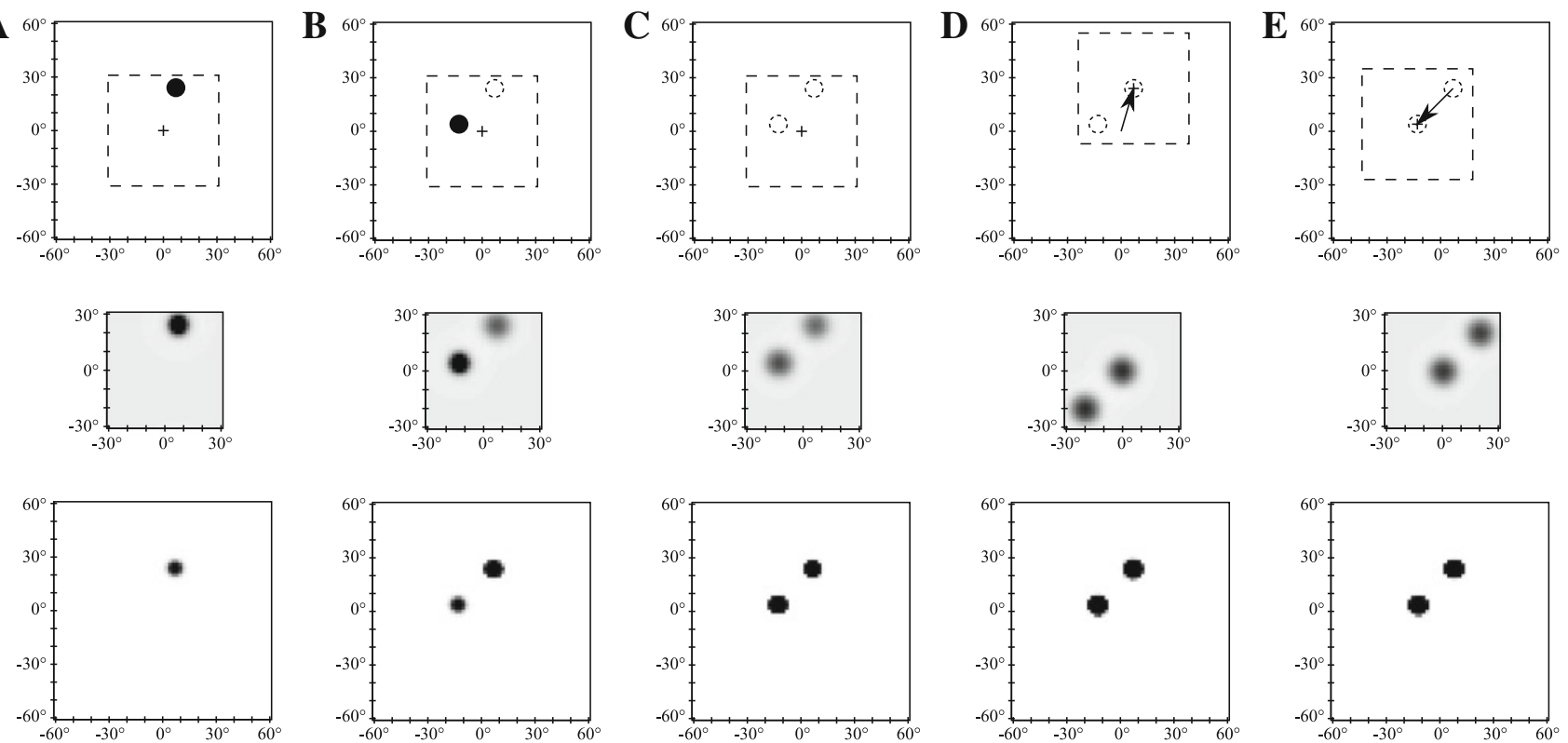

Fig. 8 Double-step saccade task. Top row Visual scene and gaze changes. The cross indicates the current fixation point, the dashed box indicates the current field of view, which yields the input to the retinocentric field. Arrows indicate gaze changes, filled circles visual stimuli, and dashed open circles show positions of stimuli that are no longer visible. Middle row Retinocentric output of the transformation field. Bottom row Output of the body-centered field (covering twice the range of the retinocentric output). A Presentation of first saccade target. B Presentation of second saccade target. C Memory representation of stimulus locations before gaze changes. D Situation briefly after the first gaze change. E Situation briefly after the second gaze change. starting at $t=400 \mathrm{~ms}$ (the saccade begins $50 \mathrm{~ms}$ later and takes $50 \mathrm{~ms}$ to complete, Fig. 8d). Another saccade signal for a gaze change to the second stimulus is given at $t=600 \mathrm{~ms}$ (Fig. 8e). In order to make a correct second saccade, the new retinocentric position of the second stimulus must be determined.

As noted in Sect. 2.4, the model does not cover the process of saccade generation itself. Previous models of saccade generation using DNFs (Kopecz and Schöner 1995; Wilimzig et al. 2006) have addressed how the metric distance between saccade targets determines whether selection or averaging occurs, including in double-step paradigms (Ottes et al. 1984; Aslin and Shea 1987). DNF models have addressed repulsion and attraction effects between metrically close items in related work on spatial working memory (Simmering et al. 2008). To concentrate on remapping, we will only use stimulus locations here that are relatively distant from each other.

In the model, the metrics for both saccades in the doublestep task can be obtained from the transformation field output, projected onto the retinocentric reference frame (analogous to what is plotted in red in Figs. 5, 6). This yields a 2D distribution in the full implementation, determined by summing the output of the 4D transformation field along the two dimensions of gaze direction. Figure 8 shows this output for each phase of the sequential saccade task (middle row), as well as the output of the body-centered field (bottom row). When the two stimuli are presented, two activity peaks form consecutively in the transformation field, and are visible in the retinocentric output at the stimulus positions (Fig. 8a, b). Activity peaks likewise form in the body-centered field for both stimuli. The body-centered positions depend also on the initial gaze direction, which was set to straight ahead for this simulation. After the two stimuli are turned off, the selfsustained activity peaks provide a memory representation of their locations (Fig. 8c).

During the first saccade, the peaks in the retinocentric field are shifted to the retinal location at which the stimulus would appear after the saccade. These peaks match, therefore, the stimulus positions in the new retinocentric reference frame (Fig. 8d). The peak that corresponds to the location of the first stimulus, which is now foveated, has been shifted to the center of the field. The position of the second peak in the retinocentric output yields the location of the second stimulus relative to the current fixation point. It therefore also provides the required metrics for the second saccade. The body-centered representation is not altered by the gaze change. When the second saccade is executed, an analogous shift in the retinocentric output takes place, now bringing the second peak to the central location (Fig. 8e). Again the body-centered representation remains unchanged.

We measured the accuracy for the metrics of the second saccade that this mechanism can provide by determining the retinocentric position of the corresponding activity peak after the first gaze shift, at time $t=600 \mathrm{~ms}$. We varied the position 
of the first saccade target between $0^{\circ}$ and $25^{\circ}$ in steps of $1^{\circ}$ both horizontally and vertically, leading to correspondingly varied metrics of the first gaze shift. The second target was always $20^{\circ}$ to the left and below the first target. The simulations produced only small deviations of the peak position from the expected remapped location, depending on the metrics of the first saccade. The mean amplitude of the error was $0.29^{\circ}$ (maximal error: $0.85^{\circ}$, standard deviation from expected location: $0.35^{\circ}$ ). Errors in the remapping occur mostly when the peak in the transformation field does not shift exactly along the input ridge from the body-centered field, which can be caused by lateral interactions in trials where old and new peak positions partly overlap. This typically results in a slight under-compensation of the gaze shift, occurring most frequently if the first saccade is of intermediate length. The remapping is also affected by the errors in the result of the gaze update described above. Possible motor errors, which produce deviations between the CD signal and the actual saccade metrics, are not taken into account in the model.

Note that the neuronal structures involved in accounting for trans-saccadic memory are the same ones discussed in the previous subsection.

\subsection{Time course of remapping}

To test whether the model can account for the experimental evidence for retinocentric remapping of Duhamel et al. (1992), we performed several simulations that emulated the experimental situations. Duhamel et al. (1992) recorded from macaque LIP neurons, which were characterized by their retinocentric receptive fields. By comparing these neuronal responses to the retinocentric output of the transformation field, we are making the assumption that the observed neurons are either gain-modulated themselves (something that was not tested in experiment) or that these neurons are driven from gain-modulated input neurons. To make the comparison, we select a single retinocentric position that matches the receptive field center of an experimentally observed neuron, and determine the time course of the retinocentric output at that position. The retinocentric output of the transformation field, read out at single position, yields an estimate for the average response of all gain-modulated neurons with a matching retinocentric receptive field. A very similar response pattern can also be expected in purely retinocentric representations that receive input from these gain-modulated neurons.

The original task did not require the macaques to memorize stimulus locations. This makes is possible that the system is operated in the "perceptual mode," in which peaks persist only while localized input is present. In other cases, the updated information may be relevant for a task and could potentially be retained by the fields. In those cases, the sys- tem should be operated in the "memory mode," in which peaks are sustained when localized input is removed. Given the limitations of the experimental record, we look at both regimes for this discussion.

Experimental and simulation results are shown in Fig. 9. All simulations were performed using the full implementation of the model for 2D inputs. In the first simulation, a constant fixation stimulus and a transient probe stimulus (located $20^{\circ}$ above and $10^{\circ}$ to the right of the fixation point and presented for $150 \mathrm{~ms}$ ) are applied the retinocentric field. Transformation field output is measured for the location of the probe stimulus. Similar to the experimental result recorded in an analogous condition (Fig. 9a), there is a steep rise in the retinocentric output of the transformation field following the stimulus presentation with a brief delay, then an initial drop followed by a slow decay of output strength after the stimulus is turned off (Fig. 9b). The prolonged activity in the transformation field is the result of stabilizing interactions within and between transformation and body-centered field, as described before. For the perceptual mode (solid line in Fig. 9b), the output strength reaches zero approximately $360 \mathrm{~ms}$ after stimulus disappearance, for the memory mode (dashed line) it stabilizes at an intermediate level and may be sustained indefinitely if not perturbed.

In a second experiment that we simulated, the probe stimulus remains active, but a saccade moves it out of the receptive field of the neuron under investigation (the visual input to this neuron is therefore the same as in the previous experiment). This is achieved by shifting the fixation point to a new location at the time of probe stimulus onset. The same stimulus settings are reproduced in the model, and a $20^{\circ}$ leftward saccade is simulated by applying an appropriate input to the saccade field, starting $100 \mathrm{~ms}$ after the shift of the fixation point. The positions of all visual stimuli in the simulation are shifted by $20^{\circ}$ rightward at the time of the saccade to emulate the effect of the gaze change. In both the original data and the simulation (Fig. 9c, d), the saccade produces a much more rapid decrease of activity than turning off the stimulus (Fig. 9a, d), and the output strength quickly reaches zeros for both the perceptual and the memory mode. In the model, this fast decrease is an effect of the changing gaze input: Through the saccade, the activity peak in the transformation field loses not only its retinocentric input but also the support from the stronger input ridge that originates from the gaze field, as the gaze direction peak moves to a new position (see Fig. 5c). Loosing both of its inputs instead of only one in the previous scenario, the peak decays much more quickly. It is important to note that in both this and the following experiment, there are additional visual stimuli present in the scene, one of them being the current saccade target. As the model allows for multi-item spatial representations and parallel remapping of multiple locations, we can account for experimental results regarding the one item under investigation while at the same 
Fig. 9 Comparison of response time courses in experimental results $(\mathbf{A}, \mathbf{C}, \mathbf{E})$ and model simulations $(\mathbf{B}, \mathbf{D}, \mathbf{F})$. For the simulation results, the time course of the transformation field output projected back onto the retinocentric reference frame is shown, for different retinocentric locations. The solid line shows the results using the perceptual mode, the dashed line shows the memory mode of the model. A Response of an LIP neuron to a visual stimulus transiently presented in its receptive field (dashed circle). B Model output at the retinocentric location of a transient visual stimulus. C Response of the same neuron to a visual stimulus that is moved out of the receptive field by a saccade. The solid vertical line indicates the beginning of the gaze change. D Output time course at the original retinocentric location of the probe stimulus for a simulated gaze change. E Neural response of a different neuron if a saccade brings the location of a previously flashed stimulus into its receptive field. F Output time course in a simulation of this scenario. The shown retinocentric location never receives direct visual input, but a previously presented stimulus would be shifted to this location by a simulated saccade. A, C, E Redrawn after Duhamel et al. (1992).
$\mathbf{A}$
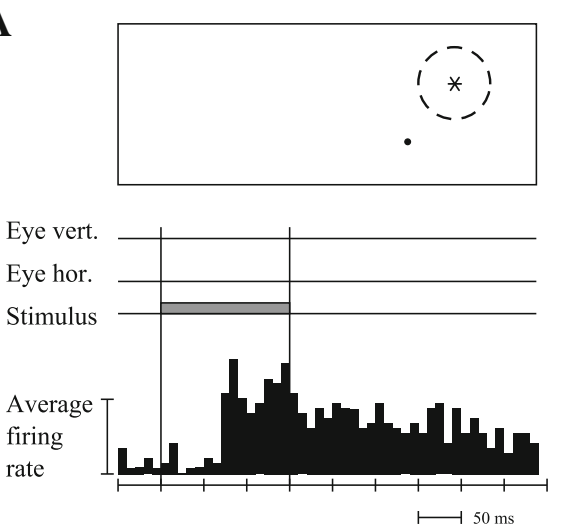

C
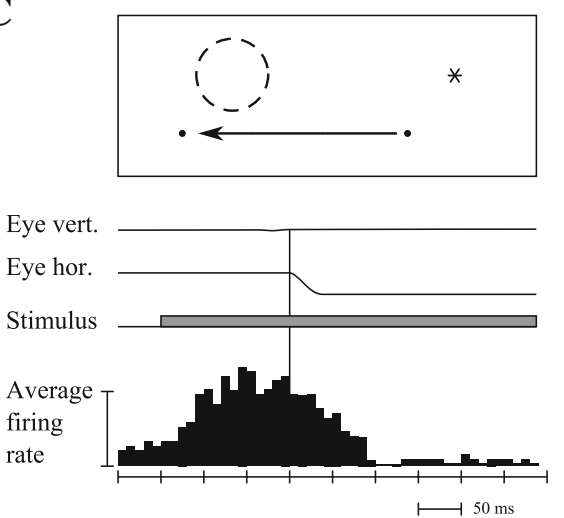

E

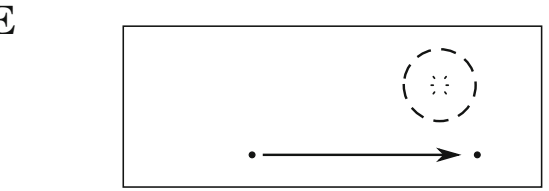

Eye vert.

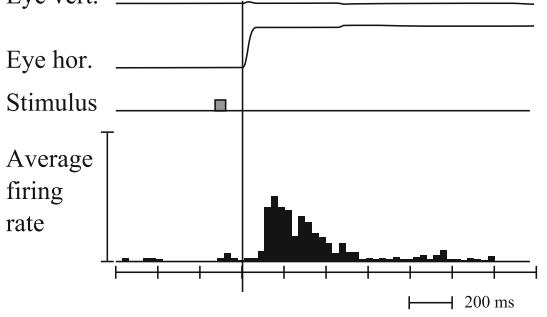

B
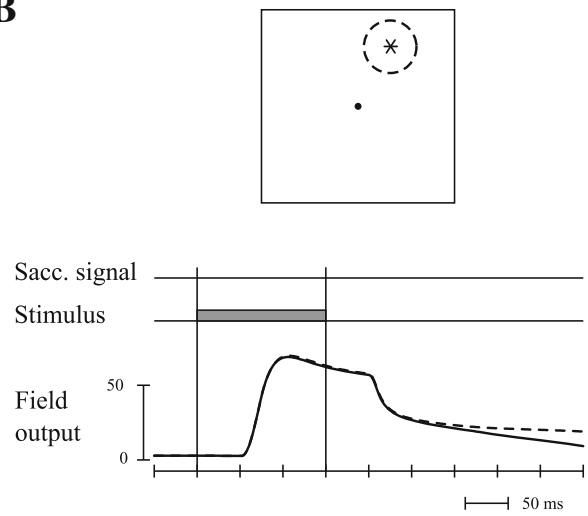

D
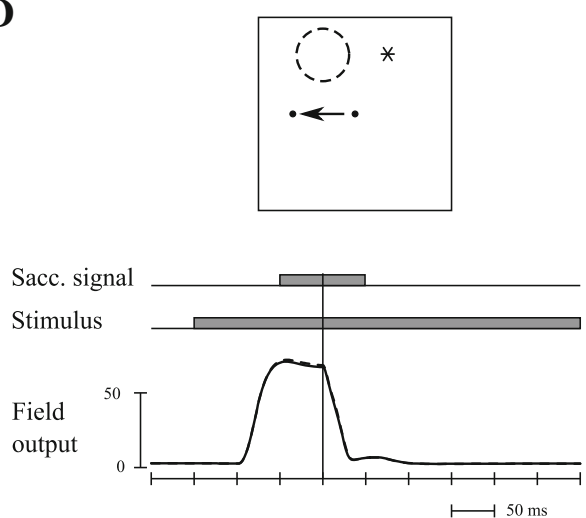

$\mathbf{F}$
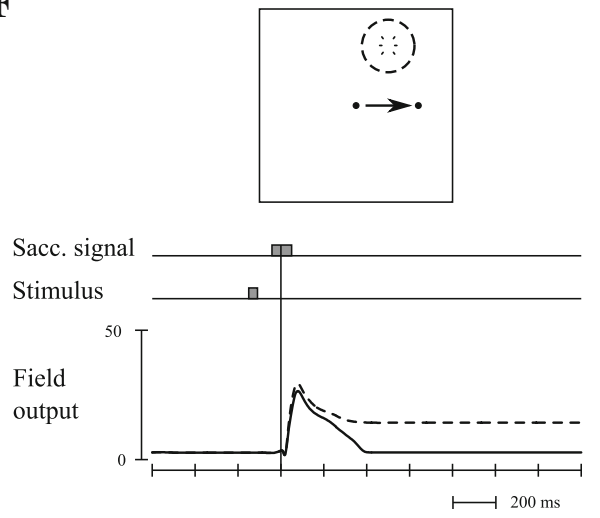

providing the required representations to plan a saccade to another stimulus.

In the third (and critical) simulation, we test whether a probe stimulus flashed briefly before a saccade is remapped to its new retinocentric location. Initially, a fixation point is presented at the center of the retinocentric field, which is then shifted to by $20^{\circ}$ to the right. At the same time, a probe stimulus is shown $20^{\circ}$ above and $10^{\circ}$ right of the center, and is extinguished again after $50 \mathrm{~ms}$. At $100 \mathrm{~ms}$ after the shift of the fixation point, an input is applied to the saccade field simulating a $20^{\circ}$ rightward saccade. The actual saccade starts $150 \mathrm{~ms}$ after the fixation point shift. Transformation field output is measured at a position $20^{\circ}$ above and $10^{\circ}$ left of the center-at a position where no stimulus is ever presented, but where the probe stimulus would appear after the saccade if it were sustained. The simulation result (Fig. 9f) reproduces the experimental signature of retinocentric remapping (Fig. 9e): Around the time of the saccade, the mechanism detailed earlier produces a sharp rise of activity at this retinocentric location, without any visual input applied to it. Depending on which mode the system is operated in, this activity either decays over a few $100 \mathrm{~ms}$ (perceptual mode), replicating the experimental findings, or it persists as a new self-sustained peak forms in the transformation field (mem- 
ory mode). The comparison of the model to experiment for this paradigm suggests that the perceptual mode is adequate. The memory mode of the model may be more appropriate for experimental studies with a memory component (e.g., Umeno and Goldberg 2001).

Note that the neuron in Fig. 9e shows remapping activity only with a substantial delay. In experiment, the latencies of different neurons vary substantially. Sommer and Wurtz (2006) report that for FEF neurons showing remapping activity, mean response onset is very close to the time of saccade initiation. The model accounts for such fast remapping. To capture the full range of variation of response onset seen in experiment would require additional assumptions (e.g., allowing for inhomogeneous connectivity patterns as in Avillac et al. 2005).

\section{Discussion}

We have shown that a dynamic neural architecture that performs reference frame transformations may account for saccadic remapping of retinocentric representations. We build on earlier DNF models of multi-item visual working memory, which represent memory items through self-sustained peaks of activity in neural populations (Johnson et al. 2008). We combine this type of representation with a mechanism for reference frame transformation that emulates the response properties of gain-modulates neurons in the parietal cortex (Zipser and Andersen 1988). Implementing that transformation mechanism within the framework of DNFs, we account for the continuous evolution of neural activation that follows visual stimulation and changes of gaze direction.

In the model, saccadic remapping within a retinocentric reference frame emerges as the result of bidirectional dynamic coupling between a retinocentric and a gaze-invariant representation. The model produces detailed time courses of activation patterns at the population level, providing quantitative fit to experimentally observed patterns of neural activity during retinocentric remapping. Such neural activity patterns have been found in largely the same cortical areas where gain modulation by gaze direction has been observed, namely LIP and FEF (Duhamel et al. 1992; Sommer and Wurtz 2006; Buneo and Andersen 2006; Cassanello and Ferrera 2007). Previously, gain modulation and remapping have been attributed to separate mechanisms (Quaia et al. 1998; Keith et al. 2010). In fact, remapping has been been interpreted as evidence against the use of gaze-invariant representations for trans-saccadic memory (Colby and Goldberg 1999; Wurtz 2008). By showing how remapping arises from a neurally plausible mechanism for reference frame transformation mechanism, we provide an interpretation of this set of results that is no longer in conflict with each other.
The model thus retains the conceptual strengths of both accounts. Representing visual information in body-centered reference frames provides robustness against the accumulation of error over multiple saccades (Karn et al. 1997), and it can be utilized for motor planning and sensor fusion (Andersen et al. 1997). The retinocentric representation enables direct attentional modulation within the predominantly retinocentric visual processing stream (Gardner et al. 2008). Retinocentric information also is relevant as input for the planning of saccadic eye movements.

We addressed the predictive nature of the experimentally observed remapping by proposing a similar neuronal account for how gaze direction is predicted. This issue has been skirted in most models of gain-modulated neurons, sometimes with the assumption that proprioceptive input provides the current gaze direction. Models of retinocentric remapping, on the other hand, have typically combined a visual or CD signal that informs about the metrics of an upcoming saccade with the retinocentric location of an item to determine its remapped location. In our approach, an internal representation of current gaze direction is predictively updated based on a CD signal. This makes it possible to achieve predictive remapping based on neurons that are gain-modulated by a gaze direction signal such as those found in LIP and FEF. At the same time, the model accounts for the observation that the remapping activity in FEF depends upon a CD transmitted from the SC via the thalamus Sommer and Wurtz (2004b, 2006).

The theoretical contribution of our model is thus to show how bidirectional coupling, operating DNFs in the regime of strong interaction, and using predictive mechanisms for both retinocentric remapping and for updating gaze direction resolves conflicts and provides a coherent interpretation of neural data. We have mapped components of the model to known neurophysiology. Not enough is known about all neural circuits involved for this mapping to be definite and final. Moreover, the level of abstraction of neural activation dynamics at the population level may not be sufficiently fine grained to capture all details of neuronal firing in the implicated brain regions. By accounting for a sizable amount of behavioral and neuronal data, however, we have highlighted signatures of the postulated principles.

\subsection{Comparison to previous models}

The general mechanism that we employ for the reference frame transformation is analogous to a number previous models of this process. The earliest was a backpropagationtrained neural network by Zipser and Andersen (1988). In an extension of this model for sequential saccades, Xing and Andersen (2000) also observed that some model neurons showed signatures of retinocentric remapping. The specific mechanism leading to this observation is substantially different from the one that we propose, using separate and func- 
tionally different populations to represent the first and the second saccade target. The remapping activity in this model is restricted to the second saccade target, it does not generalize to other stimuli and does not sufficiently account for the temporal patterns of experimentally observed remapping activity. A general strength of this class of neural network models, which use supervised learning to determine the connection weights, is that they are able to capture the heterogeneity of neural response patterns. In comparison, the DNF approach with its predefined connectivity and homogeneous representations has the advantage of being more accessible to formal analysis. It allows not only to reproduce certain experimental observations but also to disclose the precise mechanisms behind them.

Pouget and colleagues presented a number of radial basis function models that successfully account for of reference frame transformations with population codes (Pouget and Sejnowski 1997) and provide a mechanism for sensor fusion by bidirectional transformations (Denève et al. 2001; Pouget et al. 2002). These models do not, however, address saccadic remapping. Compared to these approaches to reference frame transformation, the mathematically more elaborate framework of DNFs allows a higher degree of neural realism. Specifically, the DNF model takes into account the lateral interactions within neural populations in a biologically plausible fashion. Replacing divisive normalization by local surround inhibition in particular allowed us to perform a mapping of multiple locations in parallel, a critical prerequisite in explaining the remapping data. We furthermore replaced the multiplicative combination of retinocentric position and gaze direction in these models by an additive combination with subsequent application of a sigmoid output function. Salinas and Abbott (1996) have shown that this neurally more realistic mechanism in combination with lateral interactions can account for the response properties of gain-modulated neurons.

In comparison with the two classes of models described above, the DNF approach puts special emphasis on capturing the continuous evolution of neural activity in response to changing inputs. The neural network approaches typically model visual stimuli and gaze changes as discrete events that occur and produce effects within a single simulated time step. The models of Pouget and colleagues describe the evolution of activity patterns, but are restricted to static inputs. The DNF architecture we propose aims to capture the temporal structure of both external stimuli and internal signals. Within this framework we show how macroscopically discrete events-like the abrupt shift of the retinocentric representation-emerge from the continuous change of population activity through fast transitions between qualitatively different stable states of the dynamical system.

The most comprehensive model of retinocentric remapping so far comes from Quaia et al. (1998), who explain the experimental results by a mechanism unrelated to reference frame transformations. Their model relies strongly on specific multiplicative connections between neurons, which realize all possible combinations of retinocentric position and saccade metrics. In addition, a relatively complex set of control signals is used to ensure that the shift of the retinocentric representation takes place exactly once for every saccade. More recently, Keith et al. (2010) presented a backpropagation-trained neural network that performs a direct shift of a retinocentric representation based either on the retinocentric saccade target position or an eye movement signal. While our work supplies no evidence against such a mechanism, we consider our model to offer a more parsimonious explanation for the experimental observations. It provides a detailed account for key experimental findings regarding saccadic remapping while relying only on a transformation mechanism modeled after the established response properties of gainmodulated neurons in LIP and FEF.

\subsection{Model predictions and outlook}

The central prediction from our model is that the observed shifts of retinocentric representations occur first in neurons whose responses show a modulation by gaze direction, and is triggered by a changing gaze direction signal. Xing and Andersen (2000) hinted at the existence of predictive eye position signals in LIP that might trigger the retinocentric remapping. However, while gain modulation and receptive fields are known to occur in the same cortical areas, to our knowledge no systematic investigation of the co-occurrence of these two effects on the level of single neurons has been carried out so far. Promising candidate regions to find such a co-occurrence, and thus likely sites for the integrated transformations and remapping mechanism we propose, are LIP and FEF. Based on available findings, we cannot ascertain in which of these areas the mechanism is localized (it might operate in parallel in both of them, or remapped location information might be projected from one to the other). We do however predict that the first occurrence of remapping activity will be tightly linked to gain-modulated neurons. If it can be confirmed that reference frame transformations and shifts of retinocentric representations are two aspects of the same neural mechanism, this will open up considerable synergy effects between the two lines of experiments, and will help to form a more comprehensive understanding of spatial cognition in general.

The mechanism presented here may furthermore provide a starting point to integrate another experimental observation: Deubel and colleagues (Deubel et al. 1998; Deubel 2004) found that localized stimuli which are present across a saccade are used as landmarks to estimate the metrics of the gaze shift and the remapped retinal locations of other objects. This requires matching the pre-saccadic with post- 
saccadic location information, which can be done in our system's transformation field: If a peak in the body-centered field (which has formed before the saccade) is supplied together with a peak in the retinocentric field (representing post-saccadic visual input), the intersection point of the two input ridges in the transformation field can yield an estimate of the new gaze direction. If an additional projection is added to the system that feeds this information into the gaze field, it can be used to supplement and, if necessary, correct the gaze direction estimate derived from the saccade metrics. The corrected gaze direction estimate can then provide a basis for determining the remapped locations of objects that are not visible directly after the saccade, thus explaining the experimental results. The matching process suggested here requires representations of the pre- and post-saccadically perceived object locations to be available at the same time. This is fulfilled by our mechanism, while models that propose a direct remapping without a gaze-invariant representation do not meet this requirement.

Acknowledgments The authors acknowledge support from the German Federal Ministry of Education and Research within the National Network Computational Neuroscience-Bernstein Fokus: "Learning behavioral models: From human experiment to technical assistance," grant FKZ 01GQ0951.

Open Access This article is distributed under the terms of the Creative Commons Attribution License which permits any use, distribution, and reproduction in any medium, provided the original author(s) and the source are credited.

\section{Appendix}

We give a formal description of the full implementation for 2D visual inputs and gaze directions. Each field of the architecture is identified through a unique index: $S$, saccade field; $D_{\text {hor }}$ and $D_{\text {ver }}$, $1 \mathrm{D}$ fields for the horizontal and vertical components of the gaze direction; $U_{\text {hor }}$ and $U_{\text {ver }}$, update fields for the two gaze components; $G$, combined 2D gaze field; $R, 2 \mathrm{D}$ retinocentric field; $T, 4 \mathrm{D}$ transformation field; $B, 2 \mathrm{D}$ body-centered field. The parameters of projections between two fields carry the indices of both fields, with the first index designating the target of the projection, the second its source.

All fields use the same time constant $\tau=10 \mathrm{~ms}$ and the same logistic output function (Eq. 2) with steepness parameter $\beta=4$. Interactions within and between fields are described by convolutions of field output with interaction kernels, using the $n$-dimensional convolution operation

$[f(a) * w](\mathbf{x})=\int_{R^{n}} f\left(a\left(\mathbf{x}-\mathbf{x}^{\prime}\right)\right) w\left(\mathbf{x}^{\prime}\right) \mathrm{d} \mathbf{x}^{\prime}$.
Interactions between fields typically use a simple Gaussian interaction kernel (of appropriate dimensionality $n$ ),

$w_{\text {Gauss }}(\mathbf{x})=\frac{c}{(2 \pi)^{\frac{n}{2}}|\Sigma|^{\frac{1}{2}}} \exp \left(-\frac{1}{2} \mathbf{x}^{T} \Sigma^{-1} \mathbf{x}\right)$

with connection strength $c$ and covariance matrix $\Sigma$ (if not noted otherwise, a diagonal matrix with equal entries $\sigma$ ). Lateral interactions employ either a difference of Gaussians, $w_{\text {DoG }}=w_{\text {Gauss }}^{\text {exc }}-w_{\text {Gauss }}^{\text {inh }}$ or a Gaussian excitatory kernel with a global (constant) inhibitory component, $w_{\mathrm{GI}}=$ $w_{\text {Gauss }}^{\text {exc }}-c^{\text {inh }}$. The width of the excitatory lateral interactions is $3^{\circ}$ for all fields, only for the interactions along the gaze dimensions of the transformation field we use an interaction width of $9^{\circ}$. The width of lateral inhibition in the difference-of-Gaussians kernels is always twice the width of the excitation. Other field and interaction parameters are given in Tables 1 and 2.

For simulations, fields were sampled discretely in space (at equally spaced positions at intervals of $1^{\circ}$ for the transformation module and $0.5^{\circ}$ for the gaze update module) and in time (with a temporal interval of $2 \mathrm{~ms}$ ), and the differential equations solved numerically using the Euler method. In the field equations given below, we omit the dependence of activation on time for conciseness.

Table 1 Resting levels and strengths of lateral interactions.

\begin{tabular}{llll}
\hline Field & Resting level $h$ & Lat. excitation $\left(c^{\text {exc }}\right)$ & Lat. inhibition $\left(c^{\text {inh }}\right)$ \\
\hline$S$ & -2 & 0 & 0 \\
$U$ & -2 & 10 & 0.075 \\
$D$ & 0 & 8 & 0.55 \\
$G$ & 0 & 0 & 0.075 \\
$R$ & -2 & 5 & 7.5 \\
$T$ & -2 & 7.5 & 25 \\
$B$ & -2 & 9 & 15 \\
\hline
\end{tabular}

Table 2 Parameters of projections between fields.

\begin{tabular}{lll}
\hline Projection & Strength $(c)$ & Width $(\sigma)$ \\
\hline$U S$ & 0.45 & $6^{\circ}$ \\
$U D$ & 0.7 & $6^{\circ}$ \\
$D U$ & 1.125 & $3^{\circ}$ \\
$G D^{\text {exc }}$ & 7.5 & $3^{\circ}$ \\
$G D^{\text {inh }}$ & 0.1 & (global) \\
$T G$ & 9.5 & $9^{\circ}$ \\
$T R$ & 1.2 & $3^{\circ}$ \\
$T B^{\text {exc }}$ & 1 & $3^{\circ}$ \\
$T B^{\text {inh }}$ & 0.5 & $6^{\circ}$ \\
$B T$ & 0.125 & $3^{\circ}$ \\
\hline
\end{tabular}


Gaze update module

The activation $a_{\mathrm{S}}$ of the saccade field is governed by the differential equation

$\tau \dot{a}_{\mathrm{S}}(x, y)=-a_{\mathrm{S}}(x, y)+h_{\mathrm{S}}+i_{\mathrm{S}}(x, y)$,

with an external input $i_{\mathrm{S}}$ indicating the metrics of an upcoming saccade (in retinocentric coordinates). The horizontal and vertical component of the saccade metrics are then extracted and fed as input into the separate update fields,

$$
\begin{aligned}
\tau \dot{a}_{\mathrm{U}_{\text {hor }}}(x, y)= & -a_{\mathrm{U}_{\mathrm{hor}}}(x, y)+h_{\mathrm{U}}+\left[f\left(a_{\mathrm{U}_{\mathrm{hor}}}\right) * w_{\mathrm{UU}}\right](x, y) \\
& +\left[f\left(a_{\mathrm{D}_{\text {hor }}}\right) * w_{\mathrm{UD}}\right](-x)+\left[F_{\mathrm{hor}}\left(a_{\mathrm{S}}\right) * w_{\mathrm{US}}\right] \\
& (x+y) \\
\tau \dot{a}_{\mathrm{U}_{\mathrm{ver}}}(x, y)= & -a_{\mathrm{U}_{\mathrm{ver}}}(x, y)+h_{\mathrm{U}}+\left[f\left(a_{\mathrm{U}_{\mathrm{ver}}}\right) * w_{\mathrm{UU}}\right](x, y) \\
& +\left[f\left(a_{\mathrm{D}_{\mathrm{ver}}}\right) * w_{\mathrm{UD}}\right](-x)+\left[F_{\mathrm{ver}}\left(a_{\mathrm{S}}\right) * w_{\mathrm{US}}\right] \\
& (x+y)
\end{aligned}
$$

with

$$
\begin{aligned}
& F_{\text {hor }}\left(a_{\mathrm{S}}\right)(x)=\int f\left(a_{\mathrm{S}}(x, y)\right) \mathrm{d} y \\
& F_{\text {ver }}\left(a_{\mathrm{S}}\right)(y)=\int f\left(a_{\mathrm{S}}(x, y)\right) \mathrm{d} x .
\end{aligned}
$$

The 1D gaze fields provide a second input to the update fields and in turn receive input from them by integrating over one dimension of the update field,

$$
\begin{aligned}
\tau \dot{a}_{\text {Dhor }}(x)= & -a_{\mathrm{D}_{\text {hor }}}(x)+h_{\mathrm{D}}+\left[f\left(a_{\mathrm{D}_{\text {hor }}}\right) * w_{\mathrm{DD}}\right](x) \\
& +\left[F_{\mathrm{D}}\left(a_{\mathrm{U}_{\text {hor }}}\right) * w_{\mathrm{DU}}\right](x) \\
\tau \dot{a}_{\mathrm{D}_{\text {ver }}}(x)= & -a_{\mathrm{D}_{\text {ver }}}(x)+h_{\mathrm{D}}+\left[f\left(a_{\mathrm{D}_{\text {ver }}}\right) * w_{\mathrm{DD}}\right](x) \\
& +\left[F_{\mathrm{D}}\left(a_{\mathrm{U}_{\mathrm{ver}}}\right) * w_{\mathrm{DU}}\right](x)
\end{aligned}
$$

with

$$
F_{\mathrm{D}}\left(a_{\mathrm{U}}\right)(y)=\int f\left(a_{\mathrm{U}}(x, y)\right) \mathrm{d} x .
$$

The separate gaze representations for the horizontal and the vertical component are then combined in a single $2 \mathrm{D}$ field:

$$
\begin{aligned}
\tau \dot{a}_{\mathrm{G}}(x, y)= & -a_{\mathrm{G}}(x, y)+h_{\mathrm{G}}+\left[f\left(a_{\mathrm{G}}\right) * w_{\mathrm{GG}}\right](x, y) \\
& +\left[f\left(a_{\mathrm{D}_{\mathrm{hor}}}\right) * w_{\mathrm{GD}}\right](x)+\left[f\left(a_{\mathrm{D}_{\mathrm{ver}}}\right) * w_{\mathrm{GD}}\right](y) .
\end{aligned}
$$

The interaction kernel $w_{\mathrm{GD}}$ contains a global inhibitory term in addition to the Gaussian excitatory component. Lateral interactions in the update fields and all gaze fields are of the local excitation/global inhibition type.

Transformation module

The retinocentric field is dominantly driven by external input $i_{\mathrm{R}}$, representing the locations of visually perceived objects, and is modulated by lateral interactions of the difference-ofGaussians type:

$$
\begin{aligned}
\tau \dot{a}_{\mathrm{R}}(x, y)= & -a_{\mathrm{R}}(x, y)+h_{\mathrm{R}}+i_{\mathrm{R}}(x, y)+\left[f\left(a_{\mathrm{R}}\right) * w_{\mathrm{RR}}\right] \\
(x, y) & (16)
\end{aligned}
$$

The activation of the 4D transformation field is governed by the equation:

$$
\begin{aligned}
\tau \dot{a}_{\mathrm{T}}(u, v, x, y)= & -a_{\mathrm{T}}(u, v, x, y)+h_{\mathrm{T}} \\
& +\left[f\left(a_{\mathrm{T}}\right) * w_{\mathrm{TT}}\right](u, v, x, y) \\
& +\left[f\left(a_{\mathrm{G}}\right) * w_{\mathrm{TG}}\right](u, v) \\
& +\left[f\left(a_{\mathrm{R}}\right) * w_{\mathrm{TR}}\right](x, y)+\left[f\left(a_{\mathrm{B}}\right) * w_{\mathrm{TB}}\right] \\
& (u+x, v+y) .
\end{aligned}
$$

This field likewise uses a difference-of-Gaussians for the interaction kernel. The Gaussians are wider in the dimensions of gaze direction and more narrow in the dimensions of retinocentric position. In addition, the kernel $w_{\mathrm{TT}}$ is rotated in the $u x$ - and $w y$-plane by an angle of $\phi=\frac{3}{16} \pi$ to reflect the shape of activation peaks induced by the combination of retinocentric input and body-centered input along the diagonals. Unlike the other interaction kernels between different fields, the kernel $w_{\text {TB }}$ of the difference-of-Gaussians type to limit the overall increase of activity when the body-centered representation is forming.

The body-centered field receives input from the transformation field, summed up along the diagonals, and features difference-of-Gaussians type lateral interactions:

$$
\begin{aligned}
\tau \dot{a}_{\mathrm{B}}(x, y)= & -a_{\mathrm{B}}(x, y)+h_{\mathrm{B}}+\left[f\left(a_{\mathrm{B}}\right) * w_{\mathrm{BB}}\right](x, y) \\
& +\left[F_{\mathrm{B}}\left(a_{\mathrm{T}}\right) * w_{\mathrm{BT}}\right](x, y)
\end{aligned}
$$

with

$F_{\mathrm{B}}\left(a_{\mathrm{T}}\right)(x, y)=\iint f\left(a_{\mathrm{T}}(p, q, x-p, y-q)\right) \mathrm{d} p \mathrm{~d} q$.

The resting level $h_{\mathrm{B}}$ is set to -2 for the perceptual mode and increased to -1.8 for the memory mode.

Stimulus settings

An upcoming gaze change is signaled by applying a Gaussian input with strength $c_{\mathrm{S}}^{\text {stim }}=5$ and width $\sigma_{\mathrm{S}}^{\text {stim }}=4^{\circ}$ centered at the planned saccade endpoint to the saccade field. This input has a fixed duration of $100 \mathrm{~ms}$, the actual gaze change is assumed to start $50 \mathrm{~ms}$ after its onset and take $50 \mathrm{~ms}$ to complete.

The visual stimuli are modeled as phasic-tonic Gaussian inputs to the retinocentric field. We assume a fixed delay $t_{\mathrm{R}}^{\mathrm{del}}=50 \mathrm{~ms}$ between the onset of the simulated stimulus and the start of the field input (to account for transmission times to the parietal cortex). For a single stimulus located at 
$\mathbf{p}_{\mathrm{R}}=\left[p_{\mathrm{R}, x}, p_{\mathrm{R}, y}\right]$ and present from time $t_{\mathrm{s}}$ to $t_{\mathrm{e}}$, the input $i_{\mathrm{R}}$ at any time $t$ between $t_{\mathrm{S}}+t_{\mathrm{R}}^{\mathrm{del}}$ and $t_{\mathrm{e}}+t_{\mathrm{R}}^{\mathrm{del}}$ is given by

$$
\begin{aligned}
i_{\mathrm{R}}(x, y, t)= & \left(c_{\mathrm{R}}^{\text {ton }}+c_{\mathrm{R}}^{\text {phas }} \exp \left(-t+t_{\mathrm{s}}+t_{\mathrm{R}}^{\mathrm{del}}\right)\right) \\
& \exp \left(-\frac{\left(x-p_{\mathrm{R}, x}\right)^{2}+\left(y-p_{\mathrm{R}, y}\right)^{2}}{2 \sigma_{\mathrm{R}}^{\text {stim }^{2}}}\right),
\end{aligned}
$$

with $c_{\mathrm{R}}^{\text {ton }}=2, c_{\mathrm{R}}^{\text {phas }}=5$, and $\sigma_{\mathrm{R}}^{\text {stim }}=3^{\circ}$ for all stimuli.

During a gaze change (corresponding to the last $50 \mathrm{~ms}$ of a saccade signal), all visual stimuli are turned off and the retinocentric field is globally suppressed. To this end, the input $i_{\mathrm{R}}$ is set to a constant negative value during this period, $i_{\mathrm{R}}(x, y)=c_{\mathrm{R}}^{\text {sup }} \forall x, y$, with $c_{\mathrm{R}}^{\text {sup }}=-5$.

\section{References}

Amari S (1977) Dynamics of pattern formation in lateral-inhibition type neural fields. Biol Cybern 27(2):77-87

Andersen RA, Mountcastle VB (1983) The influence of the angle of gaze upon the excitability of the light-sensitive neurons of the posterior parietal cortex. J Neurosci 3(3):532-548

Andersen RA, Essick GK, Siegel RM (1985) Encoding of spatial location by posterior parietal neurons. Science 230:456-458

Andersen RA, Snyder LH, Bradley DC, Xing J (1997) Multimodal representation of space in the posterior parietal cortex and its use in planning movements. Annu Rev Neurosci 20:303-330

Aslin RN, Shea SL (1987) The amplitude and angle of saccades to double-step target displacements. Vision Res 27(11):1925-1942

Avillac M, Denève S, Olivier E, Pouget A, Duhamel JR (2005) Reference frames for representing visual and tactile locations in parietal cortex. Nat Neurosci 8(7):941-949

Bridgeman B, Stark L (1991) Ocular proprioception and efference copy in registering visual direction. Vision Res 31(11):1903-1913

Brotchie PR, Andersen RA, Snyder LH, Goodman SJ (1995) Head position signals used by parietal neurons to encode locations of visual stimuli. Nature 375(6528):232-235

Buneo CA, Andersen RA (2006) The posterior parietal cortex: Sensorimotor interface for the planning and online control of visually guided movements. Neuropsychologia 44(13):2594-2606

Cassanello CR, Ferrera VP (2007) Computing vector differences using a gain field-like mechanism in monkey frontal eye field. J Physiol (Lond) 582:647-664

Cavanagh P, Hunt AR, Afraz A, Rolfs M (2010) Visual stability based on remapping of attention pointers. Trends Cogn Sci 14(4):147153

Cisek P (2006) Integrated neural processes for defining potential actions and deciding between them: A computational model. J Neurosci 26(38):9761-9770

Colby CL, Goldberg ME (1999) Space and attention in parietal cortex. Annu Rev Neurosci 22:319-349

Denève S, Latham PE, Pouget A (2001) Efficient computation and cue integration with noisy population codes. Nat Neurosci 4:826-831

Deubel H (2004) Localization of targets across saccades: Role of landmark objects. Vis Cogn 11(2-3):173-202

Deubel H, Bridgeman B, Schneider WX (1998) Immediate post-saccadic information mediates space constancy. Vision Res 38:31473159

Duhamel JR, Colby CL, Goldberg ME (1992) The updating of the representation of visual space in parietal cortex by intended eye movements. Science 255(5040):90-92
Eggert J, van Hemmen JL (2001) Modeling neuronal assemblies: theory and implementation. Neural Comput 13(9):1923-1974

Erlhagen W, Schöner G (2002) Dynamic field theory of movement preparation. Psychol Rev 109(3):545-572

Fazl A, Grossberg S, Mingolla E (2009) View-invariant object category learning, recognition, and search: How spatial and object attention are coordinated using surface based attentional shrouds. Cogn Psychol 58(1):1-48

Freedman EG, Stanford TR, Sparks DL (1996) Combined eye-head gaze shifts produced by electrical stimulation of the superior colliculus in rhesus monkeys. J Neurophysiol 76(2):927-952

Freedman EG, Sparks DL (1997) Eye-head coordination during headunrestrained gaze shifts in rhesus monkeys. J Neurophysiol 77(5):2328-2348

Gardner JL, Merriam EP, Movshon JA, Heeger DJ (2008) Maps of visual space in human occipital cortex are retinotopic, not spatiotopic. J Neurosci 28(15):3988-3999

Golomb JD, Marino AC, Chun MM, Mazer JA (2011) Attention doesn't slide: spatiotopic updating after eye movements instantiates a new, discrete attentional locus. Atten Percept Psychophys 73(1):7-14

Goossens HHLM, Van Opstal AJ (2006) Dynamic ensemble coding of saccades in the monkey superior colliculus. J Neurophysiol 95:2326-2341

Guthrie BL, Porter JD, Sparks DL (1983) Corollary discharge provides accurate eye position information to the oculomotor system. Science 221(4616):1193-1195

Hallett PE, Lightstone AD (1976) Saccadic eye movements to flashed targets. Vision Res 16(1):107-114

Heiser LM, Colby CL (2006) Spatial updating in area lip is independent of saccade direction. J Neurophysiol 95:2751-2767

Johnson JS, Spencer JP, Schöner G (2008) Moving to higher ground: the dynamic field theory and the dynamics of visual cognition. New Ideas Psychol 26(2):227-251

Karn KS, Møller P, Hayhoe MM (1997) Reference frame in saccadic targeting. Exp Brain Res 115(2):267-282

Keith GP, Blohm G, Crawford JD (2010) Influence of saccade efference copy on the spatiotemporal properties of remapping: A neural network study. J Neurophysiol 103(1):117-139

Kopecz K, Schöner G (1995) Saccadic motor planning by integrating visual information and pre-information on neural dynamic fields. Biol Cybern 73(1):49-60

Kusunoki M, Goldberg ME (2003) The time course of perisaccadic receptive field shifts in the lateral intraparietal area of the monkey. J Neurophysiol 89:1519-1527

Mays LE, Sparks DL (1980) Saccades are spatially, not retinocentrically, coded. Science 208:1163-1165

Nakamura K, Chung HH, Graziano MSA, Gross CG (1999) Dynamic representation of eye position in the parieto-occipital sulcus. J Neurophysiol 81(5):2374-2385

Ottes FP, Van Gisbergen JAM, Eggermont JJ (1984) Metrics of saccade responses to visual double stimuli: two different modes. Vision Res 24(10):1169-1179

Pouget A, Sejnowski TJ (1997) Spatial transformations in the parietal cortex using basis functions. J Cogn Neurosci 9(2):222-237

Pouget A, Denève S, Duhamel JR (2002) A computational perspective on the neural basis of multisensory spatial representations. Nature Rev Neurosci 3:741-747

Quaia C, Optican LM, Goldberg ME (1998) The maintenance of spatial accuracy by the perisaccadic remapping of visual receptive fields. Neural Netw 11(7-8):1229-1240

Quaia C, Lefèvre P, Optican LM (1999) Model of the control of saccades by superior colliculus and cerebellum. J Neurophysiol 82(2):999-1018

Salinas E, Abbott LF (1996) A model of multiplicative neural responses in parietal cortex. Proc Natl Acad Sci USA 93(21):11,95611,961 
Simmering VR, Schutte AR, Spencer JP (2008) Generalizing the dynamic field theory of spatial cognition across real and developmental time scales. Brain Res 1202:68-86

Snyder LH, Grieve KL, Brotchie P, Andersen RA (1998) Separate body- and world-referenced representations of visual space in parietal cortex. Nature 394(6696):887-891

Sommer MA, Wurtz RH (2004a) What the brain stem tells the frontal cortex. i. oculomotor signals sent from superior colliculus to frontal eye field via mediodorsal thalamus. J Neurophysiol 91(3):13811402

Sommer MA, Wurtz RH (2004b) What the brain stem tells the frontal cortex. ii. role of the sc-md-fef pathway in corollary discharge. J Neurophysiol 91(3):1403-1423

Sommer MA, Wurtz RH (2006) Influence of the thalamus on spatial visual processing in frontal cortex. Nature 444:374-377

Stricanne B, Andersen RA, Mazzoni P (1996) Eye-centered, head-centered, and intermediate coding of remembered sound locations in area lip. J Neurophysiol 76(3):2071-2076

Tanaka M (2007) Spatiotemporal properties of eye position signals in the primate central thalamus. Cereb Cortex 17(7):1504-1515

Thelen E, Schöner G, Scheier C, Smith LB (2001) The dynamics of embodiment: a field theory of infant perseverative reaching. Behav Brain Sci 24(1):1-34

Trappenberg TP, Dorris MC, Munoz DP, Klein RM (2001) A model of saccade initiation based on the competitive integration of exogenous and endogenous signals in the superior colliculus. J Cogn Neurosci 13(2):256-271
Umeno MM, Goldberg ME (2001) Spatial processing in the monkey frontal eye field. II. Memory responses. J Neurophysiol 86(5):2344-2352

Wang XJ (2001) Synaptic reverberation underlying mnemonic persistent activity. Trends Neurosci 24(8):455-463

White III RL, Snyder LH (2004) A neural network model of flexible spatial updating. J Neurophysiol 91(4):1608-1619

Wilimzig C, Schneider S, Schöner G (2006) The time course of saccadic decision making: dynamic field theory. Neural Netw 19(8):1059_ 1074

Wilson HR, Cowan JD (1973) A mathematical theory of the functional dynamics of cortical and thalamic nervous tissue. Biol Cybern 13(2):55-80

Wurtz RH (2008) Neuronal mechanisms of visual stability. Vision Res 48(20):2070-2089

Xing J, Andersen RA (2000) Models of the posterior parietal cortex which perform multimodal integration and represent space in several coordinate frames. J Cogn Neurosci 12(4):601-614

Zipser D, Andersen RA (1988) A back-propagation programmed network that simulates response properties of a subset of posterior parietal neurons. Nature 331(6158):679-684 